\title{
A nanoliposome-based photoactivable drug delivery system for enhanced cancer therapy and overcoming treatment resistance
}

This article was published in the following Dove Press journal:

International Journal of Nanomedicine

14 November 2017

Number of times this article has been viewed

\author{
Jinjin Shi',2 \\ Yu Su' \\ Wei Liu' \\ Junbiao Chang ${ }^{2}$ \\ Zhenzhong Zhang ${ }^{1,2}$ \\ 'School of Pharmaceutical Sciences, \\ Zhengzhou University, Zhengzhou, \\ ${ }^{2}$ Collaborative Innovation Center \\ of New Drug Research and Safety \\ Evaluation, Zhengzhou, People's \\ Republic of China
}

\begin{abstract}
Recently, stimuli-responsive drug delivery systems (DDSs) with high spatial/temporal resolution bring many benefits to cancer treatment. However, cancer cells always develop ways to resist and evade treatment, ultimately limit the treatment efficacy of the DDSs. Here, we introduce photo-activated nanoliposomes (PNLs) that impart light-induced cytotoxicity and reversal of drug resistance in synchrony with a photoinitiated and rapid release of antitumor drug. The PNLs consist of a nanoliposome doped with a photosensitizer (hematoporphyrin monomethyl ether [HMME]) in the lipid bilayer and an antitumor drug doxorubicin (DOX) encapsulated inside. PNLs have several distinctive capabilities: 1) carrying high loadings of DOX and HMME and releasing the payloads in a photo-cleavage manner with high spatial/temporal resolution at the site of actions via photocatalysis; 2) reducing drug efflux in MCF-7/multidrug resistance cells via decreasing the level of P-glycoprotein induced by photodynamic therapy (PDT); 3) accumulating in tumor site taking advantage of the enhanced permeability and retention effect; and 4) combining effective chemotherapy and PDT to exert much enhanced anticancer effect and achieving significant tumor regression in a drug-resistant tumor model with little side effects.
\end{abstract}

Keywords: drug delivery, photo-responsive release, photodynamic-chemotherapy, photocatalysis, multidrug resistance

\section{Introduction}

Chemotherapy is the current leading treatment for many types and different stages of cancer. ${ }^{1,2}$ However, most of the cytotoxic chemotherapeutic agents are limited in their clinical applications due to their serious side effects. ${ }^{3,4}$ Recently, drug delivery systems (DDSs), which increase tumor selectivity and reduce toxicity, have been receiving a lot of attention. ${ }^{5}$ However, many of the reported DDSs still need to improve. For example, the drug release in many DDSs relies on spontaneous degradation of the nanocarriers in vivo and do not allow for controlled drug release, and drugs encapsulated in the DDS could not rapidly, completely be released in the tumor site. ${ }^{2,6}$ Moreover, the traditional DDSs always suffer from unexpected drug release during circulation. ${ }^{7}$

Although several nanoscale DDSs with controlled drug release ability exhibit great success such as $\mathrm{pH}$-responsive, ${ }^{8} \mathrm{GSH}$-responsive, ${ }^{9}$ temperature-responsive, ${ }^{10}$ and light-responsive ${ }^{11-15}$ DDSs, another great challenge in oncology remains unresolved. A number of resistance mechanisms and escape pathways of tumors ultimately limit the treatment efficacy of the DDSs. ${ }^{16}$ For example, the emergence of multidrug resistance (MDR) is a main obstacle for successful treatment. ${ }^{17}$ Cancer cells often develop 
drug resistance and stop responding to chemotherapeutics after repeated sessions of chemotherapy. ${ }^{18}$ Generally, MDR is a complicated phenomenon that can cause the increased drug efflux mediated by P-glycoprotein (P-gp), a member of adenosine 5 -triphosphate-binding cassette transporters, which can actively transport a broad range of anticancer drugs across the biological membranes. ${ }^{19-21}$ Therefore, MDR is also a main obstacle for DDSs.

To overcome the obstacle, an idea DDS should not only have the controlled drug release ability but also promote the intracellular accumulation of drug in the targeted cells and maintain drug concentration in an optimum level to overcome the resistance pathways. The previous studies have shown that combining multiple conventional cancer treatment modalities such as chemotherapy and photodynamic therapy (PDT) can potentially overcome drug resistance through different mechanisms of actions to achieve enhanced anticancer efficacy. ${ }^{22,23}$ Therefore, DDSs with multiple functions are needed for tumor-specific treatment and overcome the resistance mechanisms and escape pathways of tumor. ${ }^{24}$ PDT has been developed in past decades, it is a promising modality for the management of various tumors and nonmalignant diseases, based on the application of photosensitizer (PS) and activated by a specific wavelength of light and resulted in photo damage and subsequent cell death via generating a lot of reactive oxygen species (ROS; such as hydrogen peroxide, hydroxyl radicals, and super oxides). ${ }^{25-27}$

Herein, we report photo-activated nanoliposomes (PNLs) that impart photocytotoxicity to multiple tumor compartments and reversal of MDR and enable photoinitiated, rapid release of antitumor drugs (Figure 1). As schemed in Figure 1, doxorubicin (DOX) was encapsulated in the hydrophilic inner chamber of nanoliposome, and a PS hematoporphyrin monomethyl ether (HMME) was embed in the phospholipid layer of nanoliposome. PNLs were able to entrap the DOX and HMME efficiently without visible laser irradiation. In sharp contrast, as illustrated in Figure 1, when PNLs were irradiated with a $532 \mathrm{~nm}$ laser, a large number of ROS were generated, leading to the oxidation of phospholipid layer, thereby enabling the burst release of DOX. The release of DOX could be precisely remote-controlled by laser irradiation with a high spatial/temporal resolution. More importantly, during the laser irradiation, ROS was also able to decrease the level of P-gp in cancer cells and reduce the drug efflux, thereby reversal of drug resistance. During the "on" state of PNLs (laser irradiation), a combined therapeutic effect (PDT combined with chemotherapy) was performed against tumor cells. The enhanced antitumor efficacy of PNLs was examined using MCF-7/MDR cells and drug-resistant breast tumor models.

\section{Materials and methods Materials}

DOX (20140511, purity >98\%) and HMME (20160302, purity $>98 \%$ ) were obtained from Beijing Yi-He Biotech Co. Ltd (Beijing, People's Republic of China). Dipalmitoyl-snglycero-3-phosphocholine (DPPC), cholesterol, distearoylsn-glycero-3-phosphoethanolamine-PEG (DSPE-PEG), and dimethyl sulfoxide were obtained from Sigma-Aldrich Co. (St Louis, MO, USA). Sulforhodamine B (SRB), DMEM cell culture medium, penicillin, streptomycin, fetal bovine serum (FBS), and heparin sodium were bought from Thermo Fisher Scientific (Waltham, MA, USA). 4',6-diamidino-2phenylindole (DAPI) and hematoxylin and eosin were supplied by Beyotime Biotechnology Co. Ltd (Shanghai, People's

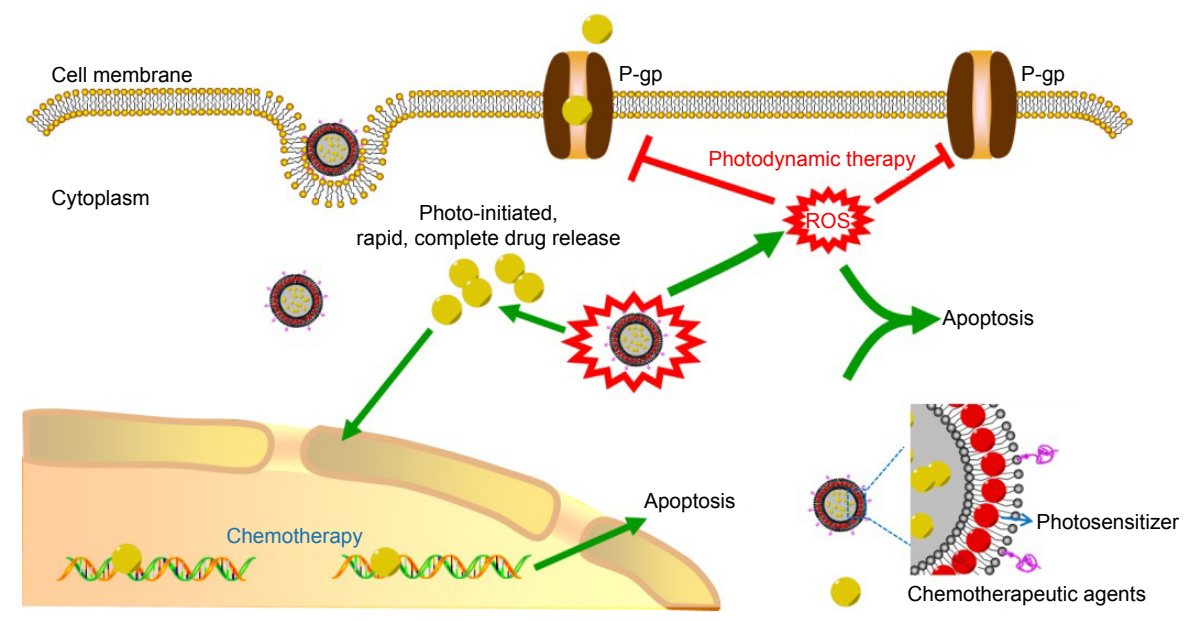

Figure I Concepts of spatiotemporal-synchronized combination therapy using PNLs.

Abbreviations: P-gp, P-glycoprotein; PNLs, photo-activated nanoliposomes; ROS, reactive oxygen species. 
Republic of China). Other reagents were acquired from China National Medicine Corporation Ltd (Chongqing, People's Republic of China). A $532 \mathrm{~nm}$ laser (diode laser, 0-300 mW/ $\mathrm{cm}^{2}, \mathrm{CW}$ ) was supplied by Changchun Laser Research Center (Changchun, People's Republic of China).

\section{Synthesis and characterization of PNLs}

Blank nanoliposome (BNL)

DPPC (40 mg), cholesterol (5 mg), and DSPE-PEG (5 mg) were dissolved in chloroform $(10 \mathrm{~mL})$, and after evaporation of chloroform, the product was dispersed in water $(4 \mathrm{~mL})$ and sonicated using an ultrasonic cell disruption system $(400 \mathrm{~W}$, 10 times). The resulting BNL nanosuspension was stored at $4^{\circ} \mathrm{C}$ in the dark until use.

\section{DOX-loaded nanoliposome (NL(DOX))}

DPPC (40 mg), cholesterol (5 mg), and DSPE-PEG (5 mg) were dissolved in chloroform $(10 \mathrm{~mL})$; after evaporation to remove the chloroform, the product was dispersed in DOX solution ( $4 \mathrm{~mL}, 1 \mathrm{mg} / \mathrm{mL}$ ). After incubation at $40^{\circ} \mathrm{C}$ for $1 \mathrm{~h}$, the product was sonicated using an ultrasonic cell disruption system (400 W, 10 times). The resulting NL(DOX) nanosuspension was stored at $4^{\circ} \mathrm{C}$ in the dark until use.

\section{HMME-loaded nanoliposome (NL(HMME))}

DPPC (40 mg), cholesterol (5 mg), DSPE-PEG (5 mg), and HMME (4 mg) were dissolved in chloroform (10 mL); after evaporation to remove chloroform, the product was dispersed in water $(4 \mathrm{~mL})$ and sonicated using an ultrasonic cell disruption system (400 W, 10 times). The resulting NL(HMME) nanosuspension was stored at $4{ }^{\circ} \mathrm{C}$ in the dark until use.

\section{PNLs}

DPPC (40 mg), cholesterol (5 mg), DSPE-PEG (5 mg), and HMME (4 mg) were dissolved in chloroform $(10 \mathrm{~mL})$; after evaporation to remove the chloroform, the product was dispersed in DOX solution ( $4 \mathrm{~mL}, 1 \mathrm{mg} / \mathrm{mL}$ ). After incubation at $40^{\circ} \mathrm{C}$ for $1 \mathrm{~h}$, the product was sonicated using an ultrasonic cell disruption system ( $400 \mathrm{~W}, 10$ times). The resulting NL(DOX) nanosuspension was stored at $4^{\circ} \mathrm{C}$ in the dark until use.

\section{Drug loading detection}

PNLs $(80 \mu \mathrm{L})$ was added to acetonitrile $(2 \mathrm{~mL})$ and then sonicated for $20 \mathrm{~min}$; after that, the sample was centrifuged and obtained the supernatant, and the amount DOX and HMME loaded in PNLs were detected by high performance liquid chromatography (HPLC) (1100; Agilent Technologies, Santa Clara, CA, USA) under the following chromatographic conditions: an Eclipse XDB-C18 column $(150 \times 4.6 \mathrm{~mm}$, $5.0 \mu \mathrm{m})$; mobile phase sodium acetate solution $(0.02 \mathrm{~mol} / \mathrm{L}) /$ acetonitrile 80:20; column temperature $40^{\circ} \mathrm{C}$; UV-Vis detector with the excitation wavelengths set at $480 \mathrm{~nm}$ (for DOX) and $395 \mathrm{~nm}$ (for HMME), respectively; flow rate $1.0 \mathrm{~mL} / \mathrm{min}$; and injection volume $20 \mu \mathrm{L}$. The encapsulation efficiency (EE) was calculated using the following formula:

$$
\mathrm{EE}=\frac{W}{W_{0}} \times 100 \%
$$

where $W$ is the weight of DOX or HMME in the nanoliposomes and $W_{0}$ is the weight of DOX or HMME initially added in liposome preparation.

\section{Characterization}

Dynamic light scattering (DLS; Zetasizer Nano ZS-90; Malvern Instruments, Malvern, UK) and transmission electron microscope (TEM; Tecnai G2 20; FEI, Memphis, TN, USA) were used for characterizing zeta potential, particle size, and morphological of BNLs, NL(DOX), NL(HMME), and PNLs.

\section{Evaluation of photoactivable ability of PNLs ROS production}

NL(DOX), NL(HMME), and PNLs (DOX: $6.6 \mu \mathrm{g} / \mathrm{mL}$, HMME: $5 \mu \mathrm{g} / \mathrm{mL}$ ) were added, and singlet oxygen sensor green (SOSG) (Thermo Fisher Scientific) was added to each well at a final concentration of $5.0 \mu \mathrm{M}$. Each experimental group contained three wells. After $532 \mathrm{~nm}$ laser $\left(100 \mathrm{~mW} / \mathrm{cm}^{2}\right)$ irradiation, a microplate reader (Spectra Max M5) was used for the acquisition of fluorescence signals, fluorescence emission at $525 \mathrm{~nm}$ was measured upon excitation at $505 \mathrm{~nm}$ using a $2.0 \mathrm{~nm}$ monochromator band pass for both excitation and emission.

\section{Drug release}

NLDOX), NL(HMME), and PNLs (DOX: $1 \mathrm{mg} / \mathrm{mL}$, HMME: $0.75 \mathrm{mg} / \mathrm{mL}$ ) were sealed in dialysis membranes (MW cutoff 12-14 kDa; Spectrapor). The dialysis bags were incubated in $10 \mathrm{~mL}$ phosphate buffer saline (PBS) buffer ( $\mathrm{pH} 7.4)$. The $532 \mathrm{~nm}$ laser $\left(300 \mathrm{~mW} / \mathrm{cm}^{2}, 15 \mathrm{~min}\right)$ irradiations were performed at $4 \mathrm{~h}$, and the released DOX or HMME was quantified by HPLC (1100).

\section{In vitro studies using MCF-7/MDR cells Cell culture}

MCF-7/MDR cells (human breast cancer cell line, P-gp highly expression, purchased commercially from Cellbio, 
(San Diego, CA, USA, Shanghai Cell Bank, item number: CBR130760, ATCC source) were cultured in normal DMEM culture medium with $10 \% \mathrm{FBS}$ and $1 \%$ penicillin/streptomycin in $5 \% \mathrm{CO}_{2}$ and $95 \%$ air at $37^{\circ} \mathrm{C}$ in a humidified incubator.

\section{Cellular uptake}

Intracellular uptake of DOX and PNLs was performed with MCF-7/MDR cells. Cells were seeded at $5 \times 10^{4}$ cells/well on glass cover slips in six-well plates. When cells reached $70 \%$ confluence, they were treated with DOX and PNLs (with the same DOX concentration: $10 \mu \mathrm{g} / \mathrm{mL}$, HMME concentration: $7.5 \mu \mathrm{g} / \mathrm{mL}$ ) in the dark for $4 \mathrm{~h}$. After staining with DAPI $(10 \mu \mathrm{g} / \mathrm{mL})$ for $15 \mathrm{~min}$, the cells were washed with PBS for three times and imaged by a Confocal Microscopy (LSM 700; Carl Zeiss Meditec AG, Jena, Germany). After removal of DOX and PNLs, MCF-7/MDR cells were incubated for another $4 \mathrm{~h}$, and for the laser irradiation group, $532 \mathrm{~nm}$ laser ( $300 \mathrm{~mW} / \mathrm{cm}^{2}, 15 \mathrm{~min}$ ) was performed at the beginning of the $4 \mathrm{~h}$; after laser irradiation, the cells were incubated in the dark for another $4 \mathrm{~h}$, and the cells were also imaged by a confocal microscopy.

\section{Detection of the level of P-gP}

MCF-7/MDR cells were treated with PNLs (DOX: $10 \mu \mathrm{g}$ / $\mathrm{mL}$, HMME: $7.5 \mu \mathrm{g} / \mathrm{mL}$ ) in the dark for $4 \mathrm{~h}$; after incubating for $4 \mathrm{~h}$, the culture medium containing PNLs was replaced by the fresh culture medium. After a $20 \mathrm{~h}$ transfection of PNLs, total protein was extracted using an M-PER protein extraction reagent (Pierce Biotech, Rockford, IL, USA) and isolated by centrifugation at $12,000 \mathrm{rpm}$ for $10 \mathrm{~min}$ at $4{ }^{\circ} \mathrm{C}$. For the laser irradiation groups, $532 \mathrm{~nm}$ laser $(300 \mathrm{~mW} /$ $\mathrm{cm}^{2}, 15 \mathrm{~min}$ ) was performed at the beginning of the $20 \mathrm{~h}$. Protein concentration was measured by a bicinchoninic acid protein assay kit (Novagen Inc., Madison, WI, USA) with bovine serum albumin as a standard. Equal amounts of protein were loaded and separated on a $12 \%$ gel by sodium dodecyl sulfate-polyacrylamide gel electrophoresis, transferred to membranes (Bio-Rad Laboratories Inc., Hercules, CA, USA) and blocked in Tris-buffered saline containing $0.1 \%$ Tween-20 and 5\% skim milk at room temperature on a horizontal shaker for $1 \mathrm{~h}$. Afterward, the membrane was probed with antibodies against P-gp. Appropriate horseradish peroxidase-conjugated antibodies (dilution 1:5,000) were used as secondary antibodies, and samples were then assayed by electrochemiluminescence (Pierce Biotech).

\section{In vitro antitumor effect}

Intracellular ROS production following laser irradiation was detected using 2,7-dichlorodi -hydrofluorescein diacetate
(DCFH-DA) Reactive Oxygen Species Assay Kit. MCF-7/ MDR cells were seeded at $5 \times 10^{4}$ cells $/$ well in six-well plates. Following incubation with PNLs (DOX: $10 \mu \mathrm{g} / \mathrm{mL}$; HMME: $7.5 \mu \mathrm{g} / \mathrm{mL}$ for $4 \mathrm{~h}$ ), DCFH-DA was loaded into the cells. After incubation for $30 \mathrm{~min}$, cells were washed twice with PBS and then exposed to $532 \mathrm{~nm}$ laser $\left(300 \mathrm{~mW} / \mathrm{cm}^{2}, 15 \mathrm{~min}\right)$ irradiation; after incubation for $1 \mathrm{~h}$, fluorescence images of treated cells were acquired using a confocal microscopy (LSM 700).

MCF-7/MDR cells were plated in 96-well plates and then incubated for $24 \mathrm{~h}$. After incubating, the medium was replaced with fresh culture medium containing free DOX (10 $\mu \mathrm{g} / \mathrm{mL}), \mathrm{NL}(\mathrm{DOX})$ (DOX: $10 \mu \mathrm{g} / \mathrm{mL})$, HMME (7.5 $\mu \mathrm{g} / \mathrm{mL}$ ), NL(HMME) (HMME: $10 \mu \mathrm{g} / \mathrm{mL}$ ), and PNLs (DOX: $10 \mu \mathrm{g} / \mathrm{mL}$; HMME: $7.5 \mu \mathrm{g} / \mathrm{mL}$ ) for $4 \mathrm{~h}$, and then, the medium was replaced with the fresh medium. After incubation for $4 \mathrm{~h}$, the cells were or were not irradiated with $532 \mathrm{~nm}$ lasers ( $300 \mathrm{~mW} / \mathrm{cm}^{2}, 15 \mathrm{~min}$ ). After incubation for another $20 \mathrm{~h}$, standard SRB assay was carried out to determine cell viabilities. The cells were also treated with different concentrations of nonloaded BNLs for $24 \mathrm{~h}$ to investigate cytotoxicity of the blank delivery system.

MCF-7/MDR cells were plated in 96-well plates and then incubated for $24 \mathrm{~h}$. After incubating, the medium was replaced with fresh culture medium containing free DOX $(10 \mu \mathrm{g} / \mathrm{mL}), \mathrm{NL}(\mathrm{DOX})$ (DOX: $10 \mu \mathrm{g} / \mathrm{mL})$, HMME (7.5 $\mu \mathrm{g} / \mathrm{mL}), \mathrm{NL}$ (HMME) (HMME: $10 \mu \mathrm{g} / \mathrm{mL}$ ), and PNLs (DOX: $10 \mu \mathrm{g} / \mathrm{mL}$; HMME: $7.5 \mu \mathrm{g} / \mathrm{mL}$ ) for $4 \mathrm{~h}$, and then, the medium was replaced with the fresh medium. After incubation for $4 \mathrm{~h}$, the cells were or were not irradiated with $532 \mathrm{~nm}$ lasers ( $300 \mathrm{~mW} / \mathrm{cm}^{2}, 15 \mathrm{~min}$ ). After incubation for another $20 \mathrm{~h}$, the cells were then trypsinized, washed with PBS, and resuspended in binding buffer $(10 \mu \mathrm{M}$ HEPEs/ $\mathrm{NaOH}, \mathrm{pH} 7.4,140 \mu \mathrm{M} \mathrm{NaCl}$, and $\left.2.5 \mu \mathrm{M} \mathrm{CaCl}_{2}\right)$. After cell density was adjusted to $1 \times 10^{6} \mathrm{cell} / \mathrm{mL}, 1 \mu \mathrm{L}$ of recombinant human anti-Annexin V-FITC and $2 \mu \mathrm{L}$ of propidium iodide were added to $100 \mu \mathrm{L}$ of cell suspension, mixed by a vortex, and incubated at room temperature in the dark for $15 \mathrm{~min}$. Finally, $400 \mu \mathrm{L}$ of binding buffer was added to the above cells and subsequently analyzed by flow cytometry (FCM, Epics XL.MCL).

\section{In vivo experiments}

\section{Xenograft tumor mouse model}

The animal studies were conducted on female BALB/C nude mice ( 20-22 g), which were purchased from Shanghai Institutes for Biological Sciences. All the mice received care in compliance with the criteria of the National Regulation on the Management of Laboratory Animals. The MCF-7/MDR 
tumor models were generated by subcutaneous injection of $2 \times 10^{6} \mathrm{MCF}-7 / \mathrm{MDR}$ cells in $0.1 \mathrm{~mL}$ saline into the right shoulder of nude mice. The mice were used when the tumor volume reached $60-100 \mathrm{~mm}^{3}$ ( 10 days after tumor inoculation). This study was approved by the Ethics Committee of Zhengzhou University.

\section{Biodistribution}

A near-infrared dye (IR783, water-soluble) was used to mark PNLs. In detail, IR783 (20 $\mu \mathrm{g})$ was added to PNLs nanosuspension (DOX: $10 \mu \mathrm{g} / \mathrm{mL}$; HMME: $10 \mu \mathrm{g} / \mathrm{mL}$ ) and then stirred for $6 \mathrm{~h}$ to obtain the IR783-marked PNLs. Excess IR783 was removed by Sephadex G-25 column (Sigma-Aldrich Co.). A sample of $0.2 \mathrm{~mL}$ of PNLs-IR783 was intravenously injected into tumor-bearing mice, and the whole body fluorescence imaging was performed at $0.5,1,2$, $4,8,12$, and 24 after injection using a small animal imaging system (Xtreme; Bruker AXS Inc., Madison, WI, USA).

\section{In vivo antitumor effect}

MCF-7/MDR tumor-bearing mice were divided into seven groups (six mice per group), minimizing the differences of weights and tumor sizes in each group. The mice were administered with 1) no treatment, 2) DOX (5 mg/ $\mathrm{kg}, 0.2 \mathrm{~mL}$ ), 3) NL(DOX) (DOX: $5 \mathrm{mg} / \mathrm{kg}, 0.2 \mathrm{~mL}), 4)$ NL(HMME) + laser (HMME: $3.25 \mathrm{mg} / \mathrm{kg}, 0.2 \mathrm{~mL}, 532 \mathrm{~nm}$, $300 \mathrm{~mW} / \mathrm{cm}^{2}, 15 \mathrm{~min}, 4 \mathrm{~h}$ postinjection), 5) $\mathrm{NL}(\mathrm{DOX})+$ $\mathrm{NL}(\mathrm{HMME}$ ) + laser (for NL(DOX), DOX: $5 \mathrm{mg} / \mathrm{kg}, 0.2 \mathrm{~mL}$; for NL(HMME), HMME: $3.25 \mathrm{mg} / \mathrm{kg}, 0.2 \mathrm{~mL} ; 532 \mathrm{~nm}$, $300 \mathrm{~mW} / \mathrm{cm}^{2}, 15 \mathrm{~min}, 4 \mathrm{~h}$ postinjection), 6) PNLs (DOX: $5 \mathrm{mg} / \mathrm{kg}$, HMME: $3.25 \mathrm{mg} / \mathrm{kg}, 0.2 \mathrm{~mL}$ ), and 7) PNLs + laser (DOX: $5 \mathrm{mg} / \mathrm{kg}$, HMME: $3.25 \mathrm{mg} / \mathrm{kg}, 0.2 \mathrm{~mL}, 532 \mathrm{~nm}$, $300 \mathrm{~mW} / \mathrm{cm}^{2}$, $15 \mathrm{~min}, 4 \mathrm{~h}$ postinjection) were intravenously injected into mice via the tail vein every 2 days, respectively. The $532 \mathrm{~nm}$ laser irradiation was applied every time after administration. The mice were observed daily for clinical symptoms, and the tumor sizes were measured by a caliper every other day and calculated as the volume $=$ (tumor length) $\times(\text { tumor width })^{2} / 2$. After treatment for 15 days, the mice were sacrificed to collect tumor for HE staining, TUNEL staining, and P-gp immunofluorescent staining. Morphological changes of hematoxylin \& eosin (HE) staining were observed under a microscope, the images of TUNEL staining and P-gp immunofluorescent staining were acquired using a confocal microscopy (LSM 700).

\section{In vivo biosafety}

On the 16th day after the treatment of PNLs, the mice were sacrificed and blood samples were collected into tubes containing $1.5 \mathrm{mg} / \mathrm{mL} \mathrm{K}_{2}$ EDTA for complete blood cell count. Plasma was separated into cryotubes and store at $-80^{\circ} \mathrm{C}$ until blood chemistry analysis.

\section{Statistical analysis}

Quantitative data are expressed as mean \pm SD and analyzed by use of Student's $t$-test. $P$-values $<0.05$ were considered statistically significant.

\section{Results}

Preparation and characterization of PNLs

PNLs were synthesized with the lipophilic PS HMME formulated within the lipid bilayer of a liposome encapsulating a water-soluble drug DOX (Figure 2). The EE of the water-soluble drug DOX in NL(DOX) and PNLs were $\sim 43.5 \%$ and $\sim 58.8 \%$, respectively. The EE of the lipophilic PS HMME in NL(HMME) and PNLs was $\sim 69.8 \%$ and $\sim 43.7 \%$, respectively (Figure 2D and E). The particle size and zeta potential of PNLs were detected through DLS. The DLS results showed that PNLs had the average size of $126.6 \mathrm{~nm}$, the zeta potential was $-8.19 \mathrm{mV}$ (Figures 2B and $\mathrm{C}$ and $\mathrm{S} 1$ ) in water, and the average size of PNLs did not show significant change in cell medium (Figure S2). This result was also confirmed by TEM (Figures 2 and S3).

\section{Photo-activated ROS generation and drug release}

Photo-activated ROS generation

We investigated the ${ }^{1} \mathrm{O}_{2}$ production of PNLs. ${ }^{1} \mathrm{O}_{2}$ generation was determined by the fluorescence signal of a ${ }^{1} \mathrm{O}_{2}-$ responsive probe, SOSG. The results of ${ }^{1} \mathrm{O}_{2}$ production in different cases are shown in Figure 3B. Without laser irradiations, no significant generation of ${ }^{1} \mathrm{O}_{2}$ was observed in all groups, while in the cases of NL(HMME) and PNLs, a large amount of ${ }^{1} \mathrm{O}_{2}$ was generated under laser irradiation, showing that both NL(HMME) and PNLs could generate ${ }^{1} \mathrm{O}_{2}$ under laser irradiating and had a laser irradiation timedependent manner.

\section{Photocatalytic drug release}

Together with the high efficacy of ${ }^{1} \mathrm{O}_{2}$ production capacity of PNLs upon laser radiation and the efficient oxidation ability of ${ }^{1} \mathrm{O}_{2}$, PNLs was further expected to show precisely controlled drug release. To test this idea, the drug release of PNLs was investigated and the results are shown in Figure 3. As can be seen from Figure 3D, in the case of PNLs group, before laser irradiating (before $4 \mathrm{~h}$ ), the release of DOX from PNLs was very slow. While a rapid increase in the release of DOX from 

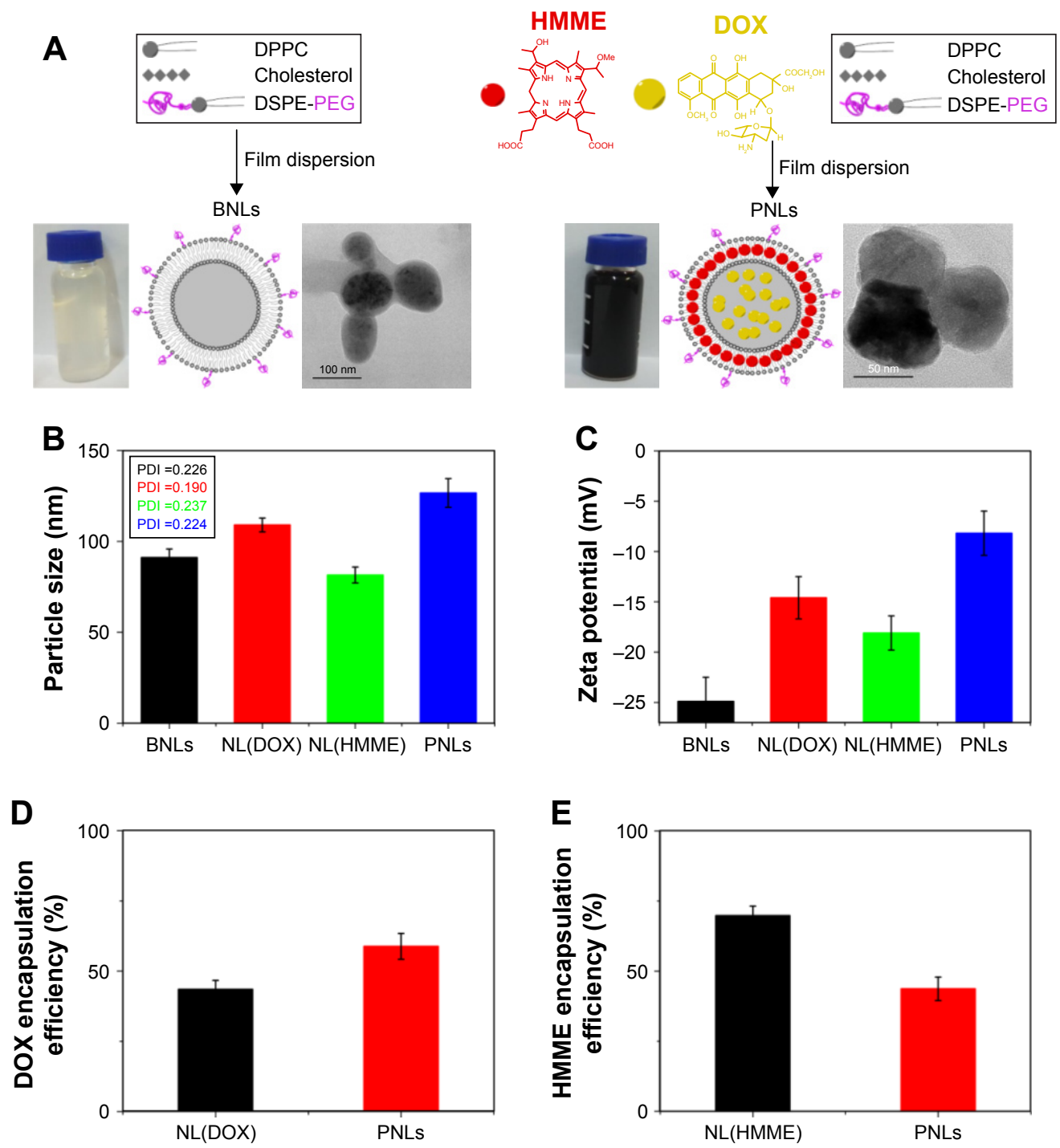

Figure 2 Preparation and characterization of PNLs.

Notes: (A) Diagrams, exteriors, and TEM images of BNLs and PNLs. (B) Particle size of BNLs, NL(DOX), NL(HMME), and PNLs. (C) Zeta potential of BNLs, NL(DOX), NL(HMME), and PNLs. (D) DOX encapsulation efficiency of NL(DOX) and PNLs. (E) HMME encapsulation efficiency of NL(HMME) and PNLs. Data presented are mean \pm SD $(n=3)$.

Abbreviations: BNLs, blank nanoliposomes; DOX, doxorubicin; DPPC, dipalmitoyl-sn-glycero-3-phosphocholine; DSPE-PEG, distearoyl-sn-glycero-3-phosphoethanolaminePEG; HMME, hematoporphyrin monomethyl ether; NL(DOX), DOX-loaded nanoliposome; NL(HMME), HMME-loaded nanoliposome; PNLs, photo-activated nanoliposomes; TEM, transmission electron microscope.

PNLs occurred upon $532 \mathrm{~nm}$ laser irradiation $\left(300 \mathrm{~mW} / \mathrm{cm}^{2}\right.$, $15 \mathrm{~min})$. Before laser irradiation, in the first $4 \mathrm{~h}$, the total released DOX was only 3.9\%. After laser irradiation for $2 \mathrm{~h}$ (from 4 to $6 \mathrm{~h}$ ), the cumulative release of loaded DOX increased from $3.9 \%$ to $88.9 \%$, while only $3.5 \%$ of DOX (from $88.9 \%$ to $92.4 \%$ ) was released during the subsequent $2 \mathrm{~h}$ (from 6 to $8 \mathrm{~h}$ ). In the case of NL(DOX) group, whether laser irradiation was performed or not, the release of DOX had no significant difference, showing that the release of DOX in NL(DOX) group was not influenced by laser irradiation. The cumulative release of DOX in PNLs without laser irradiation group was $6.4 \%$. In sharp contrast, the cumulative release of DOX in PNLs + laser group was $92.4 \%$. The above results indicated that the release of DOX in PNLs could be controlled via laser irradiation and the same results were observed in the HMME release profiles (Figure 3E). In the case of PNLs group, in the first $4 \mathrm{~h}$ (before laser irradiation), the cumulative release of HMME was $9.1 \%$. After laser irradiation for $2 \mathrm{~h}$ (from 4 to $6 \mathrm{~h}$ ), the cumulative release of HMME was $51.6 \%$ (from $9.1 \%$ to $51.6 \%$ ), while without laser irradiation, from 4 to $6 \mathrm{~h}$, the cumulative release of HMME was only $10.9 \%$ (from $9.4 \%$ to $10.9 \%$ ). The cumulative release of HMME in PNLs without laser irradiation group was $12.8 \%$ (Figure 3E). In sharp contrast, the cumulative release of DOX in PNLs + laser group 

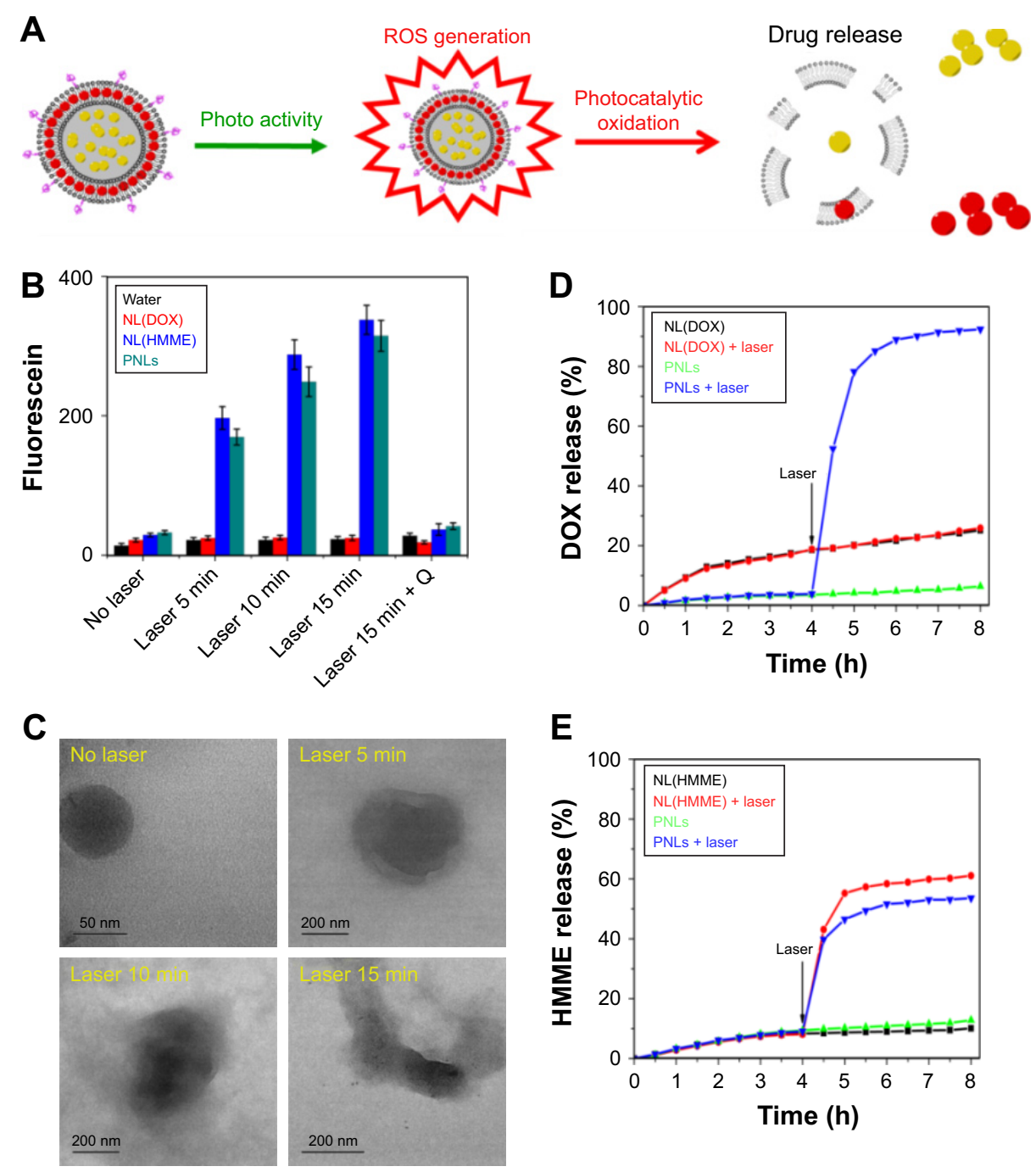

Figure 3 Photo-activated PNLs.

Notes: (A) Diagrams of PNLs upon laser irradiation. (B) ' $\mathrm{O}_{2}$ generation by PNLs (DOX: $13.3 \mu \mathrm{g} / \mathrm{mL}$, HMME: $10 \mu \mathrm{g} / \mathrm{mL}$ ) under $532 \mathrm{~nm}$ laser $\left(300 \mathrm{~mW} / \mathrm{cm}^{2}\right.$ ) for different irradiation times. (C) TEM images of PNLs without $532 \mathrm{~nm}$ laser $\left(300 \mathrm{~mW} / \mathrm{cm}^{2}\right)$ irradiation, with $532 \mathrm{~nm}$ laser $\left(300 \mathrm{~mW} / \mathrm{cm}^{2}\right)$ irradiation for $5 \mathrm{~min}$, with $532 \mathrm{~nm}$ laser $\left(300 \mathrm{~mW} / \mathrm{cm}^{2}\right)$ irradiation for $10 \mathrm{~min}$ and with $532 \mathrm{~nm}$ laser $\left(300 \mathrm{~mW} / \mathrm{cm}^{2}\right)$ irradiation for $15 \mathrm{~min}$. (D) DOX release from PNLs and NL(DOX), a $532 \mathrm{~nm}$ laser $\left(300 \mathrm{~mW} / \mathrm{cm}^{2}, 15 \mathrm{~min}\right)$ was performed at $4 \mathrm{~h}$. (E) HMME release from PNLs and NL(HMME), a $532 \mathrm{~nm}$ laser $\left(300 \mathrm{~mW} / \mathrm{cm}^{2}, 15 \mathrm{~min}\right)$ was performed at $4 \mathrm{~h}$. Data presented are mean \pm SD ( $\left.\mathrm{n}=6\right)$.

Abbreviations: DOX, doxorubicin; HMME, hematoporphyrin monomethyl ether; NL(DOX), DOX-loaded nanoliposome; NL(HMME), HMME-loaded nanoliposome; PNLs, photo-activated nanoliposomes; ROS, reactive oxygen species; TEM, transmission electron microscope.

was $53.6 \%$. According to the results of DOX and HMME release, an obvious photo-activated drug release controlled by $532 \mathrm{~nm}$ laser was also observed.

To further understand the photo-activated drug release ability of PNLs, the morphological changes of PNLs upon $532 \mathrm{~nm}$ laser irradiation were also investigated. PNLs had an obvious ball-like structure, and the phospholipid layer could be clearly observed (Figure 3C). When PNLs were irradiated by $532 \mathrm{~nm}$ laser $\left(300 \mathrm{~mW} / \mathrm{cm}^{2}\right)$ for $5 \mathrm{~min}$, PNLs became much larger and the phospholipid layer became blurred (Figure 3C). After 10 min of laser irradiation, PNLs lost its spherical structure gradually, and after $15 \mathrm{~min}$ of laser irradiation, PNLs completely lost its spherical structure, and the collapse of PNLs was also observed. After laser irradiation, PNLs had a huge morphological change and this also confirmed the photo-activated property of PNLs.

\section{Cellular PNLs internalization and intracellular release}

To examine the cellular internalization of PNLs and the intracellular release of drug, the red fluorescence of DOX within resistant MCF-7/MDR cells was observed by CLSM after incubation with DOX and PNLs for different periods of time. The results are shown in Figure 4. As expected, in the resistant MCF-7/MDR cells, the sign of free DOX was too weak to be detected even after $4 \mathrm{~h}$ of incubation, due to 

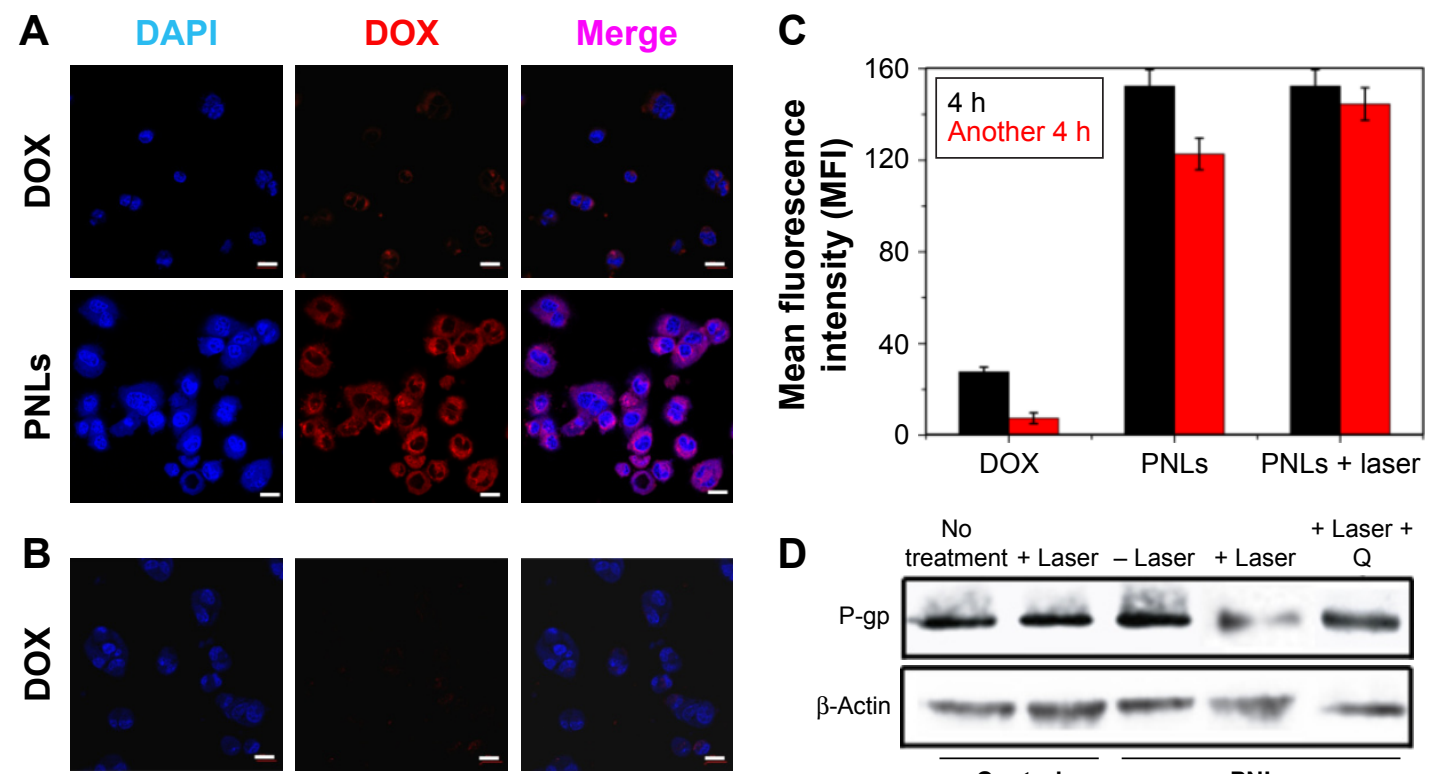

D
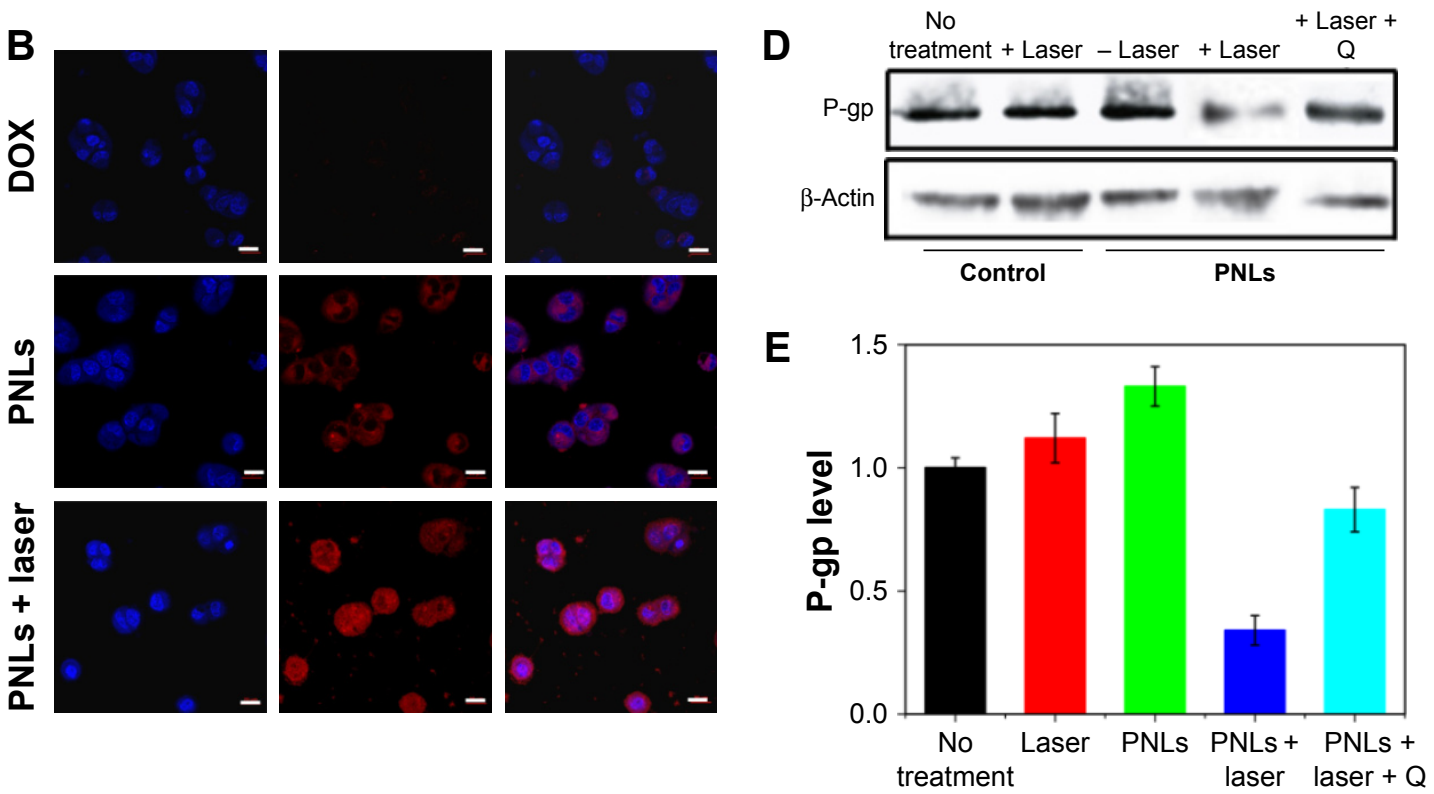

Figure 4 Cellular uptake and P-gP levels of PNLs treated cells.

Notes: (A) Internalization and retaining of DOX in MCF-7/MDR cells after treatment with DOX and PNLs for $4 \mathrm{~h}$ measured by confocal microscopy. (B) Internalization and retaining of DOX in MCF-7/MDR cells after treatment with DOX and PNLs for another $4 \mathrm{~h}$ of incubation after the removal of DOX and PNLs measured by confocal microscopy, a $532 \mathrm{~nm}$ laser irradiation $\left(300 \mathrm{~mW} / \mathrm{cm}^{2}, 15 \mathrm{~min}\right.$ ) was performed at the beginning of the $4 \mathrm{~h}$. (C) MFI of DOX and PNLs in MCF-7/MDR cells. (D) Western blotting results of P-gP expression by different treatments, for laser irradiation groups: a $532 \mathrm{~nm}$ laser irradiation was performed after $4 \mathrm{~h}$ of incubation with PNLs, Q: sodium azide (a ROS quencher, $0.5 \mathrm{mg} / \mathrm{mL}$ ). (E) Quantification of P-gP expression - normalized to $\beta$-actin and relative to the no treatment control group (normalized to I), Q: a ROS-specific scavenger, sodium azide, $2 \mu \mathrm{g} / \mathrm{mL}$. Data presented are mean $\pm \mathrm{SD}(\mathrm{n}=3)$. (A and $\mathbf{B})$ Scale bar represents $20 \mu \mathrm{m}$.

Abbreviations: DOX, doxorubicin; MDR, multidrug resistance; MFI, mean fluorescence intensity; P-gp, P-glycoprotein; PNLs, photo-activated nanoliposomes; ROS, reactive oxygen species.

the secretion of P-gp. However, in the PNLs group, a large amount of DOX was observed in MCF-7/MDR cells, indicating that PNLs could carry DOX into the cells. It is noteworthy that almost all of DOX was observed in cytosol and no DOX was observed in cell nucleus (Figure 4A), suggesting that PNLs could not enter into the cell nucleus. As we know, the intracellular DOX in the cytosol could rapidly transport to the nucleus and avidly bound to the chromosomal DNA ${ }^{2}$; this result also suggested that DOX was not released from PNLs in MCF-7/MDR cells.

To further investigate the intracellular release of drug in MCF-7/MDR cells, another $4 \mathrm{~h}$ of incubation was performed after removal of DOX and PNLs. The results are shown in Figure 4B. In the case of free DOX group, after another $4 \mathrm{~h}$ of incubation, almost none of DOX was observed (Figure 4B), demonstrating the drug efflux effect of P-gp. In the case of PNLs without laser irradiation group, the signal of DOX could be still observed in cytosol but was not as much as $4 \mathrm{~h}$ ago (Figure 4B and C). These results suggested that a small part of DOX was released form PNLs, and the released DOX was discharged out by cells via drug efflux effect of P-gp. Most of the DOX was not released from PNLs, still in cytosol not in cell nucleus. In sharp contrast, a large amount of DOX was observed in cell nucleus in the case of PNLs with 532 laser irradiation (300 mW/ $\mathrm{cm}^{2}, 15 \mathrm{~min}$ ) group. As we know, most of DOX could be released from PNLs after laser irradiation and the released DOX was not discharged out by cells but transported to the nucleus. More importantly, compared to $4 \mathrm{~h}$ ago, the signal of DOX did not show significant decrease in the case of PNLs with laser irradiation group (Figure 4B and C). 
To figure out why the released DOX was not discharged out via drug efflux effect of P-gp, the amount of P-gp in MCF-7/MDR cells influenced by PNLs with laser irradiation was further investigated. The results are shown in Figure 4D and E. Compared to the no treatment group, the expression of P-gp in the laser irradiation alone group did not show any significant decrease. As predicted, in the case of PNLs without laser irradiation group, the expression of P-gp did not show significant decrease too. In sharp contrast, the expression of P-gp had a significant decrease in PNLs with laser irradiation group, while this phenomenon could be stopped by the ROS scavenger, indicating that during laser irradiation, PNLs could generate a large amount of ROS, and the ROS could further affect the expression of P-gp, thereby reducing the efflux of free DOX in MCF-7/MDR cells.

\section{In vitro antitumor efficacy}

In this study, PNLs served not only as a drug carrier for photoactivated drug delivery but also as a PS for PDT. To investigate the PDT efficacy of PNLs, intracellular ROS induced by PNLs under $532 \mathrm{~nm}$ laser irradiating was determined. The intracellular ROS productions of PNLs with or without laser irradiation were observed by using DCFH-DA fluorescent probe staining (Figure 5A). Compared to no laser irradiation group, a large amount of DCFH signals (green fluorescence) were observed in the case of PNLs + laser group, indicating that PNLs with laser irradiation could significantly increase the intracellular ROS level in MCF-7/MDR cells.

To further evaluate the therapeutic efficacy of PNLs, standard SRB assay was conducted. Cytotoxicity of the BNL is an important consideration for its in vivo application. The cytotoxicity of BNLs is shown in Figure 5B. According to the results, BNLs had extremely small cytotoxic to MCF-7/ MDR cells.

The in vitro cytotoxicity of PNLs with or without laser irradiation was then evaluated. The results are shown in Figure 5C. In the case of laser irradiation alone group, the cell viability was $98.7 \% \pm 3.2 \%$, indicating that the MCF-7/MDR cells were not influenced by laser irradiation. Compared to DOX group $(86.2 \% \pm 2.7 \%$ ), the cell viability of NL(DOX) group showed a significant decrease $(78.3 \% \pm 3.1 \%)$, suggesting the enhanced cellular uptake effect media by liposomes. The same results were found in HMME-related groups (HMME and NL(HMME) groups). After $24 \mathrm{~h}$ of incubation, the cell viability of PNLs was $70.9 \% \pm 1.9 \%$, while after laser irradiation for $15 \mathrm{~min}$, the cell viability of PNLs significantly decreased to $16.3 \% \pm 4.6 \%$, indicating an enhanced cellkilling effect of PDT and DOX. MCF-7/MDR cell apoptosis results are shown in Figure 5D, as expected, the therapy with multimechanisms (PNLs + laser) leads to more apoptosis. After laser irradiation, on one hand, a large amount of generated ROS and released DOX from PNLs led to more cell death than the other groups; on the other hand, the generated ROS could inhibit the drug efflux effect of P-gp, thereby reducing the efflux of free DOX in MCF-7/MDR cells; therefore, PNLs + laser group led to more apoptosis than the other groups.

\section{In vivo studies of PNLs}

The in vivo biodistribution of PNLs in MCF-7/MDR tumorbearing mice was evaluated by an near infrared (NIR) fluorescence imaging system. In this study, an NIR dye IR783 was used to mark PNLs. The results are shown in Figure 6A. The fluorescence signals of IR783 and PNLs were in the highly vascularized tissues (liver and spleen) at the early time points. Compared to the IR783 group, with the passage of time, the fluorescence signals of PNLs group in tumor significantly increased and achieved a maximum at $4 \mathrm{~h}$ postinjection. In the case of PNLs group, more fluorescence signals were observed in tumor site than that of IR 783 group even at $24 \mathrm{~h}$ postinjection (Figure 6A). The results showed the tumor-targeting ability of PNLs, and after injection of PNLs for $4 \mathrm{~h}$, the accumulation of PNLs in tumor site achieved a maximum; thereby, the laser irradiation was performed at $4 \mathrm{~h}$ postinjection of PNLs.

We extended the studies from in vitro to in vivo to investigate the regression efficacy in tumor growth, which was monitored in terms of tumor volume change (Figure 6B). In the case of no treatment group, the mice were closely monitored for the continuous growth of tumor, which grew $\sim 5$ times larger on day 15 than it was initially. In the cases of DOX, NL(DOX) and NL(HMME) + laser groups, the tumors grew $>\sim 3$ times larger than they were initially after treatment for 15 days. Compared to the other groups, the tumors grew much slower in the cases of NL(DOX) + NL(HMME) + laser and PNLs + laser, indicating a significant synergistic antitumor effect of PDT and chemotherapy. Compared to PNLs without laser irradiating group, a significant decrease in tumor size was observed in PNLs + laser group, suggesting the photo-activated ability of PNLs in vivo. After treatment for 15 days, the residual tumor of the above seven groups is also shown in Figure 6C. Compared to the no treatment group $(100.0 \% \pm 17.9 \%)$, the residual tumor in DOX group $(83.6 \% \pm 11.4 \%)$ had no significant difference, indicating the resistance of the MCF-7/MDR cells. In the case of NL(DOX)+ NL(HMME) + laser group, the percentage of residual tumor was $31.7 \% \pm 4.6 \%$, much lower than that of NL(DOX) and NL(HMME) group. In the case of PNLs group, minor suppression of the tumors was seen as the percentage of residual tumor was $62.9 \% \pm 2.8 \%$ after 15 days. Contrarily, 

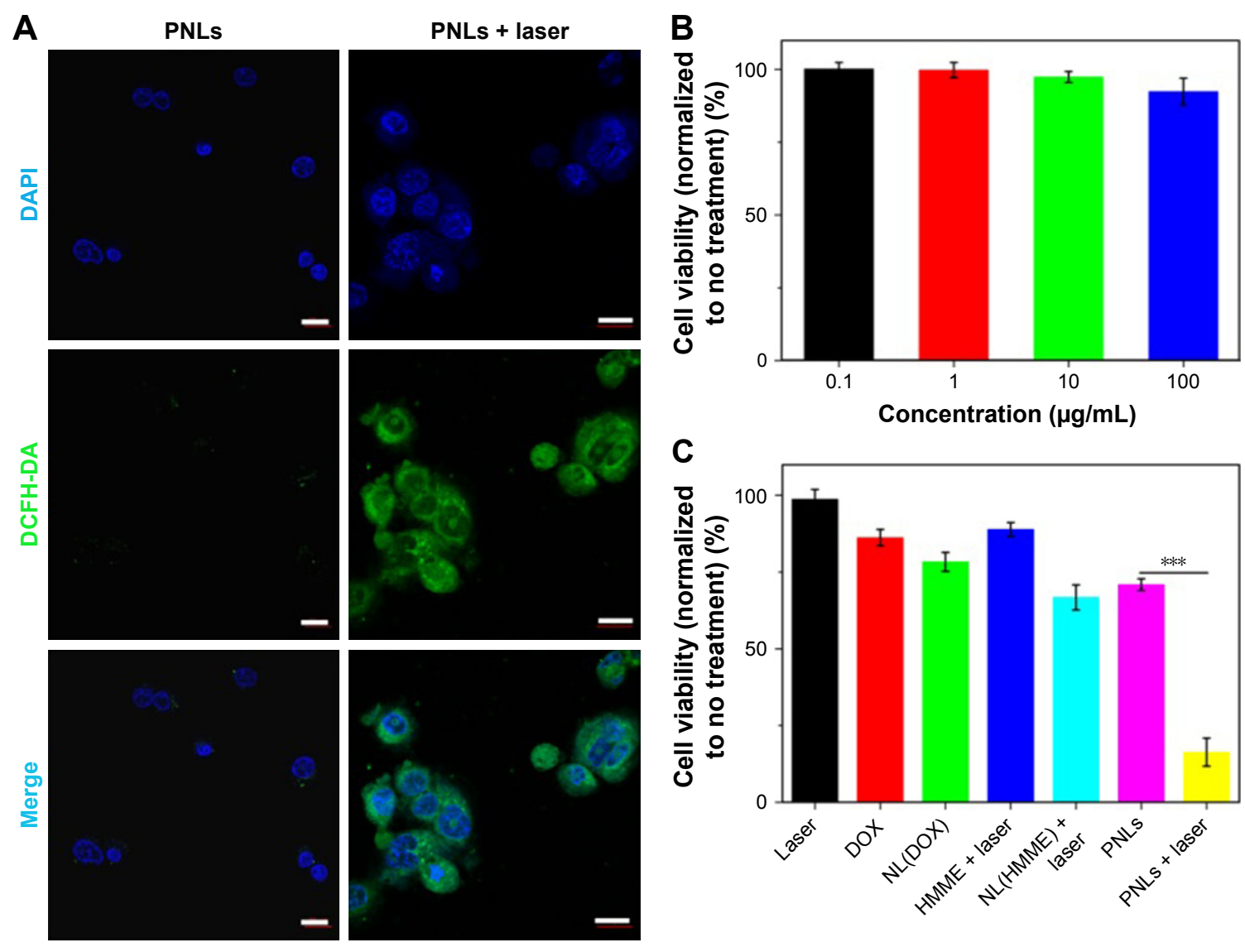

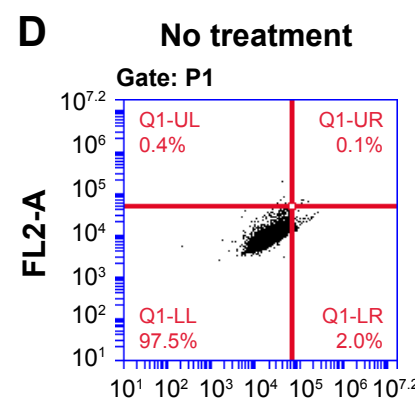

FL1-A

\section{HMME + laser}

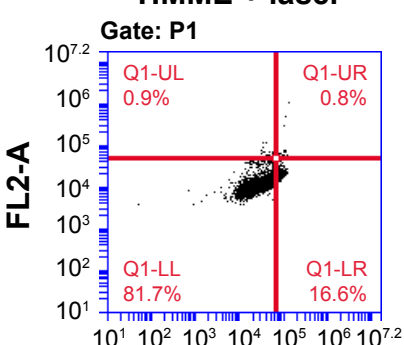

FL1-A

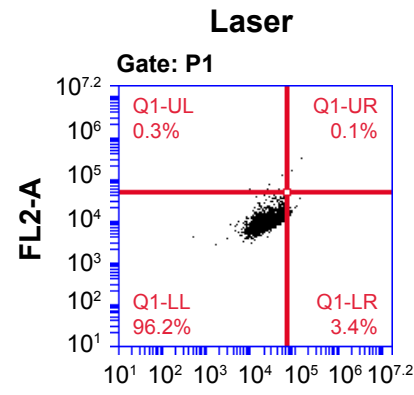

FL1-A

\section{NL(HMME) +}

laser

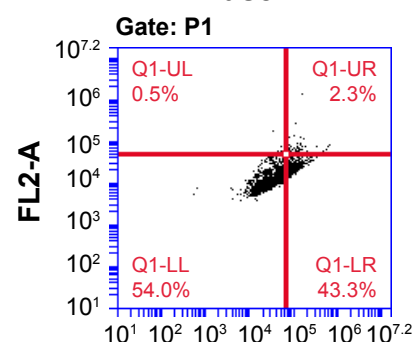

FL1-A

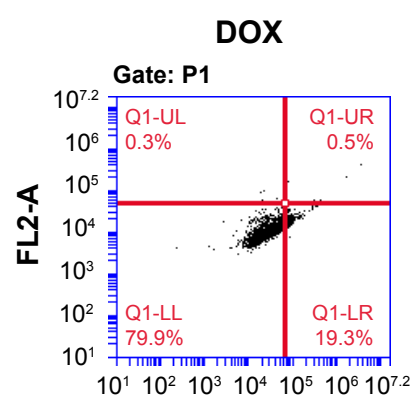

FL1-A

PNLs

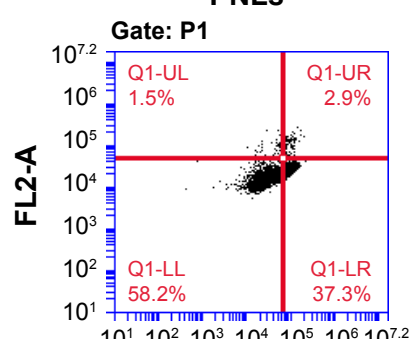

FL1-A

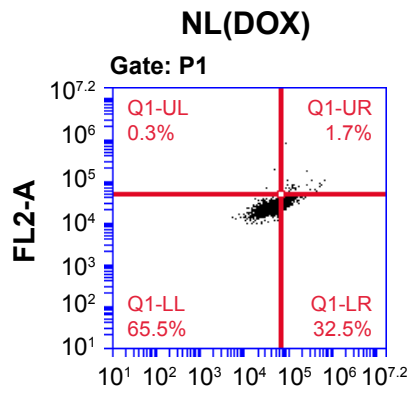

FL1-A

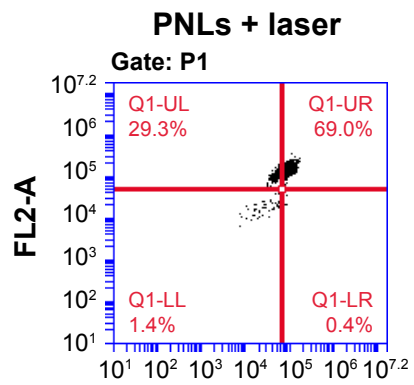

FL1-A

Figure 5 In vitro antitumor efficacy of PNLs.

Notes: (A) Detection of intracellular ROS by DCFH-DA staining in MCF-7/MDR cells; scale bar represents $20 \mu \mathrm{m}$. (B) Cytotoxicity of the BNL ( $\mathrm{n}=6$ ). (C) Cell viability of different treatments on MCF-7/MDR cells incubation for $24 \mathrm{~h}(\mathrm{n}=6)$. (D) Apoptosis in MCF-7/MDR cells of different treatments. Data presented are mean \pm SD. $* * * P<0.0$, PNLs vs PNLs+laser.

Abbreviations: DOX, doxorubicin; HMME, hematoporphyrin monomethyl ether; MDR, multidrug resistance; NL(DOX), DOX-loaded nanoliposome; NL(HMME), HMMEloaded nanoliposome; PNLs, photo-activated nanoliposomes; ROS, reactive oxygen species. 
A
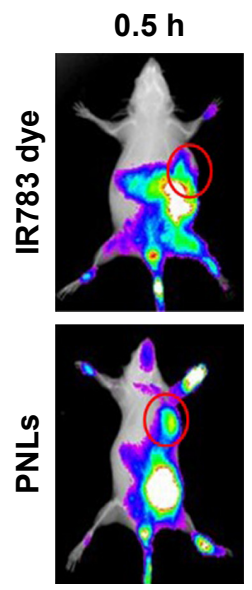

B

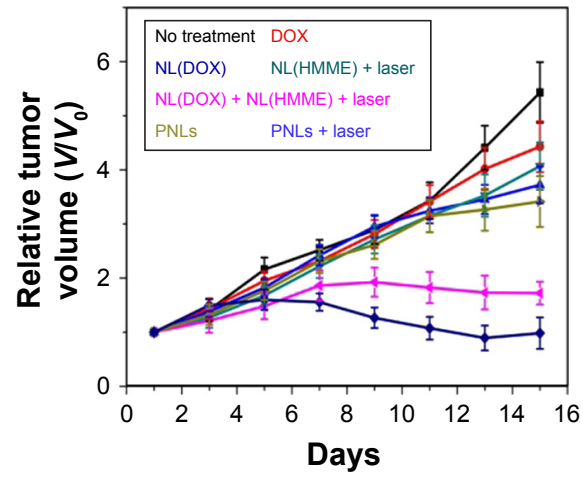

$2 \mathrm{~h}$
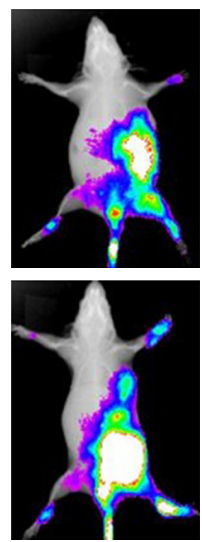
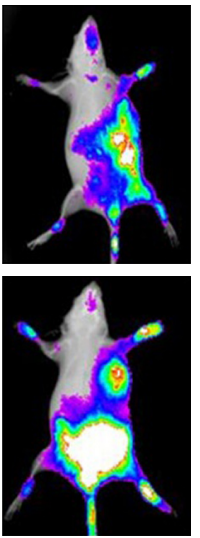

C

$4 \mathrm{~h}$

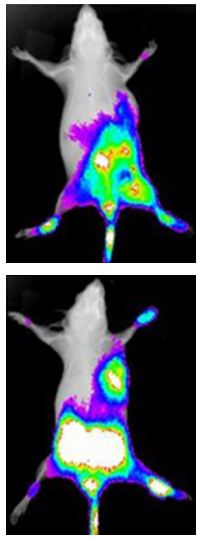

Day 25 (15 days posttreatment)
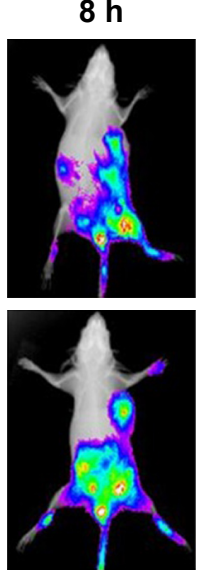
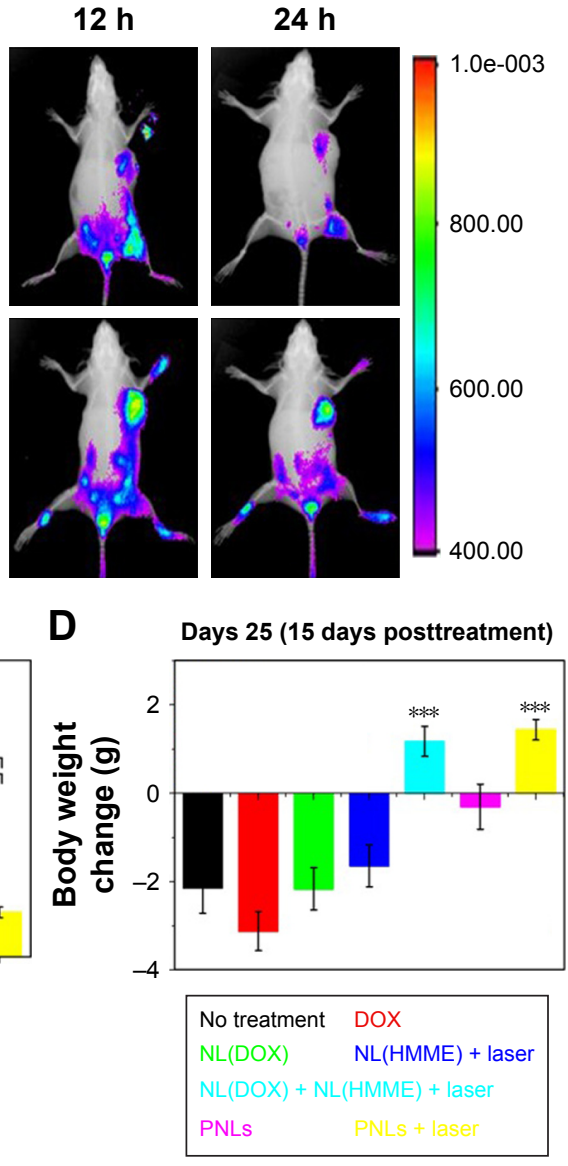

Figure 6 In vivo studies of PNLs.

Notes: (A) NIR imaging of tumor-bearing mice intravenously injected with free IR783 solution and IR783-loaded PNLs at 0.5, I, 2, 4, 8, I2, and 24 h postinjection; the red circle shows the tumor site. (B) Tumor growth of mice in different treatment groups within 15 days $(n=6)$. (C) The percentage of fraction residual tumor in different treatment groups on day 25 (I5 days posttreatment, $n=6$ ). $* * * P<0.0$ I, NL(DOX) and NL(HMME) vs NL(DOX) $+\mathrm{NL}(\mathrm{HMME})+$ laser; $\mathrm{PNLs}$ vs $\mathrm{PNLs}+$ laser; $* \mathrm{P}<0.05$, $\mathrm{NL}(\mathrm{DOX})+\mathrm{NL}(\mathrm{HMME})+$ laser vs PNLs + laser. (D) Body weight change of mice receiving different treatments $(\mathrm{n}=6)$. ${ }^{* * * P}<0.01, \mathrm{NL}(\mathrm{DOX})+\mathrm{NL}(\mathrm{HMME})+$ laser and $\mathrm{PNLs}+$ laser vs no treatment. Data presented are mean \pm SD.

Abbreviations: DOX, doxorubicin; HMME, hematoporphyrin monomethyl ether; NL(DOX), DOX-loaded nanoliposome; NL(HMME), HMME-loaded nanoliposome; PNLs, photo-activated nanoliposomes.

in PNLs + laser group, the percentage of residual tumor was only $18.1 \% \pm 2.2 \%$, and the tumor growth was totally inhibited by PNLs with laser irradiation, indicating the obvious photo-activated ability of PNLs resulted in the effectiveness of combination treatment (PDT and chemotherapy) and reversing the drug resistance, thus suppression of tumor growth. The therapeutic efficacy of PNLs was also evaluated by HE staining (Figure 7A). Compared to the no treatment group, the tumor-bearing mice treated with PNLs + laser displayed extensive cell death in the tumor tissues (Figure 7A). The HE staining results are consistent with the in vivo antitumor effects. TUNEL assay was also performed to examine apoptosis level in tumors from mice receiving different treatments. TUNEL assay showed that apoptotic nuclei were dark brown stained and normal nuclei were blue stained (Figure 7A). Compared to the no treatment group $(\sim 3.7 \%)$, NL(DOX)-treated tumor and $\mathrm{NL}(\mathrm{HMME})+$ laser-treated tumor showed $\sim 44.6$ and $~ 37.1 \%$ of apoptotic cells (Figure 7A and B). Meanwhile, high apoptosis level ( $96.7 \%)$ was observed in PNLs + laser tumor (Figure 7A and B). TUNEL staining results clearly confirmed the treatment efficacy of PNLs + laser by inducing apoptosis of tumor cells.

To further understand the antitumor effect of PNLs with laser irradiation, the P-gp level in tumor of the treatment mice was also investigated. The P-gp level in tumor tissue was examined via immunofluorescence assay, and the results are shown in Figure $7 \mathrm{C}$. In the case of no treatment and PNLs without laser irradiation group, high levels of P-gp were observed, while the P-gp level in PNLs + laser group showed a significant decrease (Figure 7C). To semiquantify the P-gp (green fluorescence) in tumors, Image-pro 
A
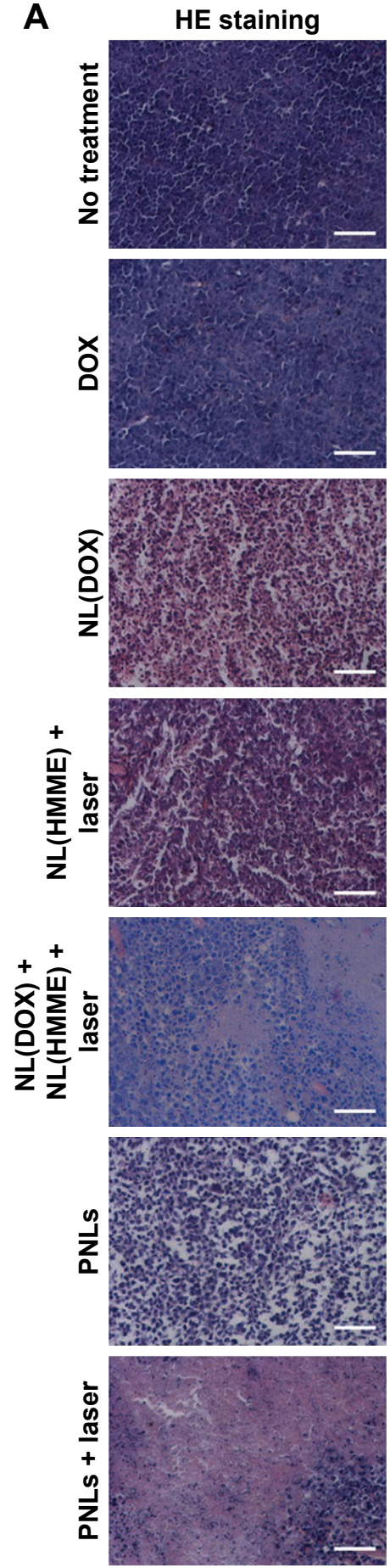

TUNEL assay
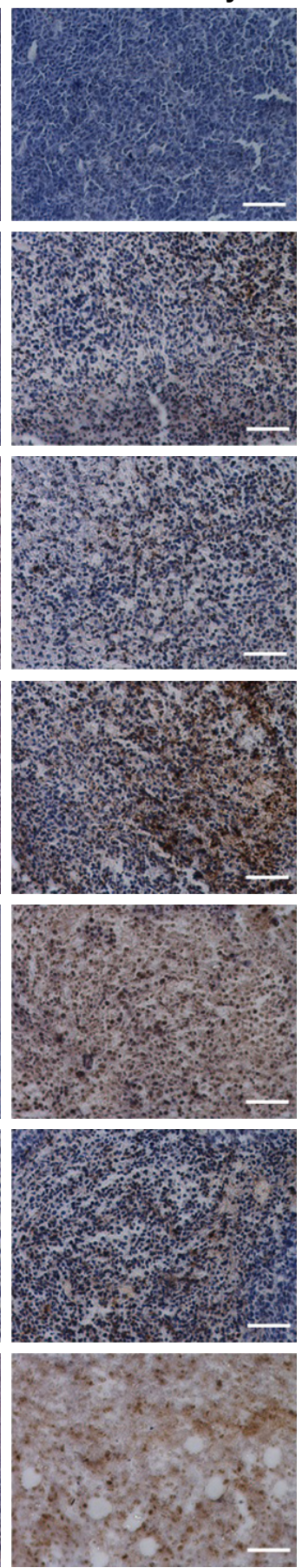

B
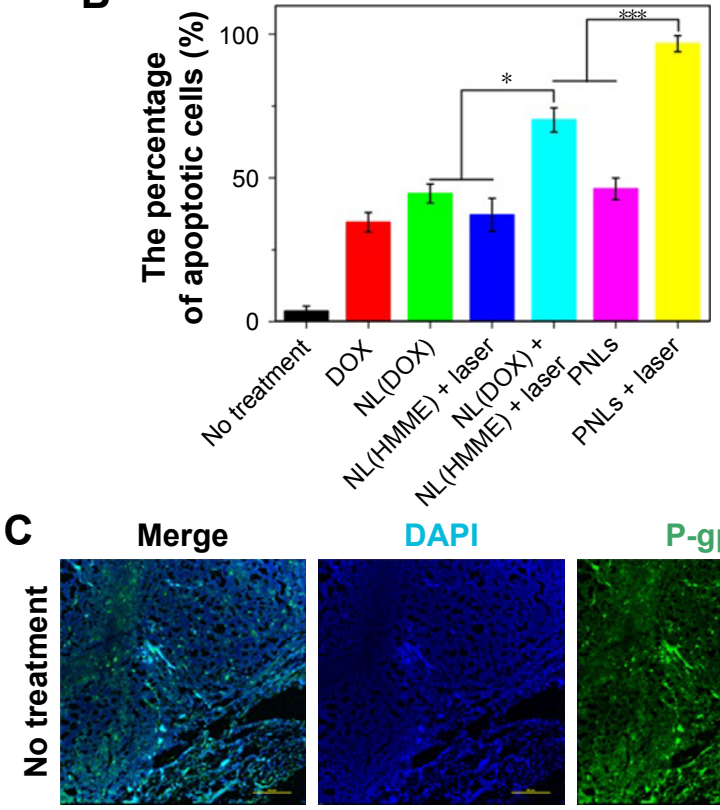

DAPI
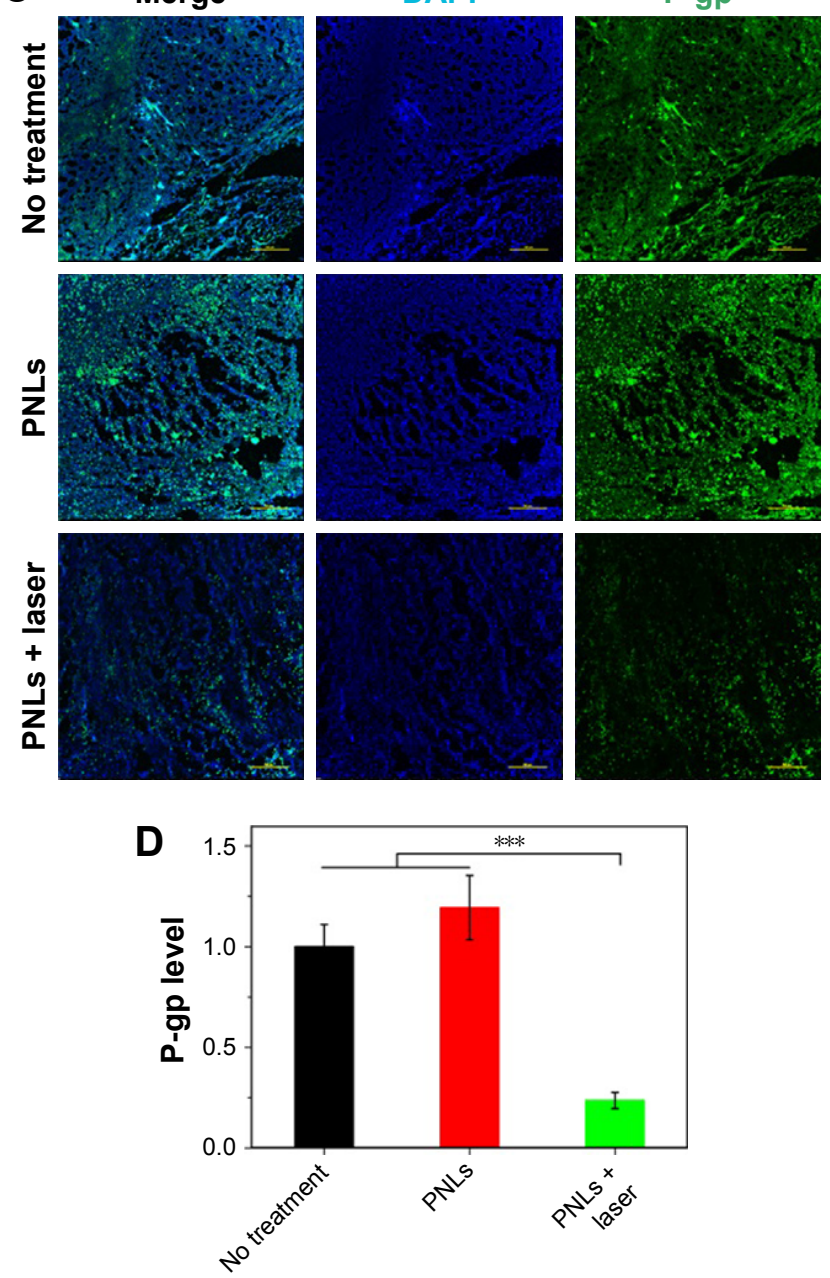

Figure 7 Ex vivo analysis of the histological characteristics of tumor.

Notes: (A) HE and TUNEL staining images of tumor tissues in mice with different treatments for 15 days; scale bar represents $200 \mu \mathrm{m}$. (B) The percentage of apoptotic cells in tumor tissues with different treatments for 15 days $(n=3)$. $* * * P<0.0 I, N L(D O X)+N L(H M M E)+$ laser and $P N L s$ vs $P N L s+$ laser; $* P<0.05$, NL(DOX) and $\mathrm{NL}(\mathrm{HMME})+$ laser vs NL(DOX) + NL(HMME) + laser. (C) P-gP immunofluorescent staining images of tumor tissues in PNLs and PNLs + laser-treated mice; scale bar represents $200 \mu \mathrm{m}$. (D) Quantification of $\mathrm{P}$-gP expression in tumor tissues - normalized to the no treatment control group (normalized to I, $\mathrm{n}=3$ ). ${ }^{* * *} \mathrm{P}<0.0 \mathrm{I}$, no treatment and PNLs vs PNLs + laser. Data presented are mean \pm SD.

Abbreviations: DOX, doxorubicin; HMME, hematoporphyrin monomethyl ether; NL(DOX), DOX-loaded nanoliposome; NL(HMME), HMME-loaded nanoliposome; P-gP, P-glycoprotein; PNLs, photo-activated nanoliposomes.

plus 6.0 was used to analyze the level of P-gp by integrated optical density of fluorescence image (Figure 7D). PNLs-treated tumor showed a higher level of P-gp than that of no treatment group, indicating that drug stimulation could increase the expression of P-gp. In sharp contrast, after treatment for 15 days, the level of P-gp in PNLs with laser irradiation group significantly decreased, suggesting that the PDT could decrease the expression of P-gp, and 
this result was consistent with the in vitro P-gp level studies (Figure 7E).

\section{In vivo biosafety}

DOX has a severe cardio toxicity; thereby, the cardio toxicity of DOX and PNLs was investigated via TUNEL staining assay. TUNEL assay showed that apoptotic nuclei were dark brown stained and normal nuclei were blue stained (Figure 8A). Compared to the no treatment group, a large amount of apoptotic cells were observed in heart tissue in DOX group. Contrarily, in the case of PNLs + laser group, almost no apoptotic cells were observed and had no significant difference with the no treatment group. These results showed that PNLs could significantly decrease the side effects of free DOX, especially the cardiotoxicity, thus improving the therapeutic window of DOX.

Potential in vivo toxicity has always been a great concern in the development of nanodrugs. Besides measuring body weights of mice in each cohort (Figure 8B), the complete blood panel data of red blood cells, white blood cells, platelet count, the blood levels of total protein, alkaline phosphatase, blood urea nitrogen, creatinine, and hemoglobin from PNLs + laser group and control group were also detected. The results are shown in Figure 8B. No observable toxicity was noted in blood analysis. Furthermore, biosafety of PNLs was also evaluated by HE staining (Figure S4). According to the results, after treatment for 15 days, no significant damage in the main organs of tumor-bearing mice was observed in the PNLs + laser group than in the no treatment group, indicating the high biosafety of PNLs with laser irradiation. All these results demonstrated that PNLs + laser show high biosafety for the combination tumor treatment presenting no significant side effects to the MCF-7/MDR tumor-bearing mice.

\section{Discussion}

Despite advances in DDSs, the stimuli-responsive controlled release DDSs with high spatial/temporal resolution are still the best choice. ${ }^{28,29}$ Among the stimuli-responsive controlled release DDSs, light-induced release DDSs have been attracted more and more attention due to their high spatial/ temporal resolution in vivo. ${ }^{30-32}$ Herein, in this study, a PNL was designed and synthesized. Compared to the reported photo-activated DDSs with complex synthesis process, ${ }^{33,34}$ the preparation of the liposome-based PNLs is very simple. As we know, liposome is the most popular vector in current drug delivery due to its mature and simple preparation, high drug loadings, prolonged blood circulation half-lives, tumortargeting ability, and high in vivo biosafety. ${ }^{35-37}$ Recently, several formulations of liposome-based DDSs have been approved by Food and Drug Administration and European Medicines Agency for the treatment of cancer. ${ }^{38}$ Thus, in this study, DPPC, DSPE-PEG (common phospholipid material), and DOX (a water-soluble antitumor drug) were used to build a DOX-loaded liposome via film dispersion method. To obtain the photo-responsive ability of the liposome-based DDS, an efficient PS HMME (water-insoluble) was inserted into the lipid layer of the DOX-loaded liposome. Take advantage of the effective ROS production of HMME under light irradiation and the strong catalytic oxidation ability of ROS, ${ }^{39}$ the prepared liposome has a photo-cleavage regular drug release. Compared to the reported temperature-responsive liposome, $<50 \%$ of drug was released when the system temperature was $>42{ }^{\circ} \mathrm{C}$ for a long time; $;^{40,41}$ in this study, $\sim 80 \%$ of the loaded drugs were released after only $15 \mathrm{~min}$ of laser irradiation $\left(300 \mathrm{~mW} / \mathrm{cm}^{2}\right)$ in the photo-cleavage liposome.

In addition, after repeated treatments with chemotherapeutic agents, tumor cells always develop strategies to increase their resistance to chemotherapy. ${ }^{17}$ Combination therapy, such as the combination of chemotherapy and PDT, can promote synergism between different treatment modalities, overcome drug resistance through distinct mechanisms of actions, and enhance the anticancer efficacy. ${ }^{42}$ In this study, upon laser irradiation, a large amount of ROS was generated from the DDS, combined with the released DOX, a synergistic therapeutic effect (PDT and chemotherapy) was expected to overcome the drug resistance, thereby improving the antitumor efficacy of the resistant tumor. Herein, a PNL (photo-controlled

A
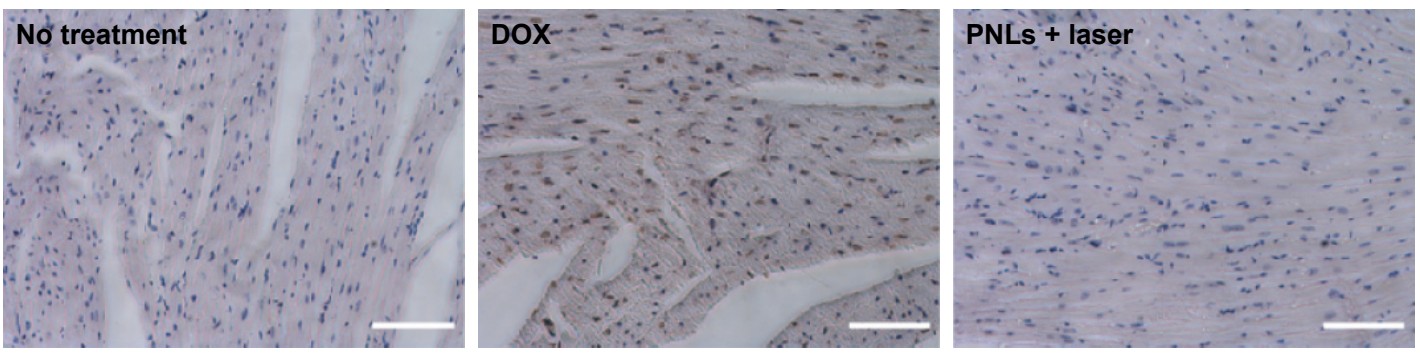

Figure 8 (Continued) 

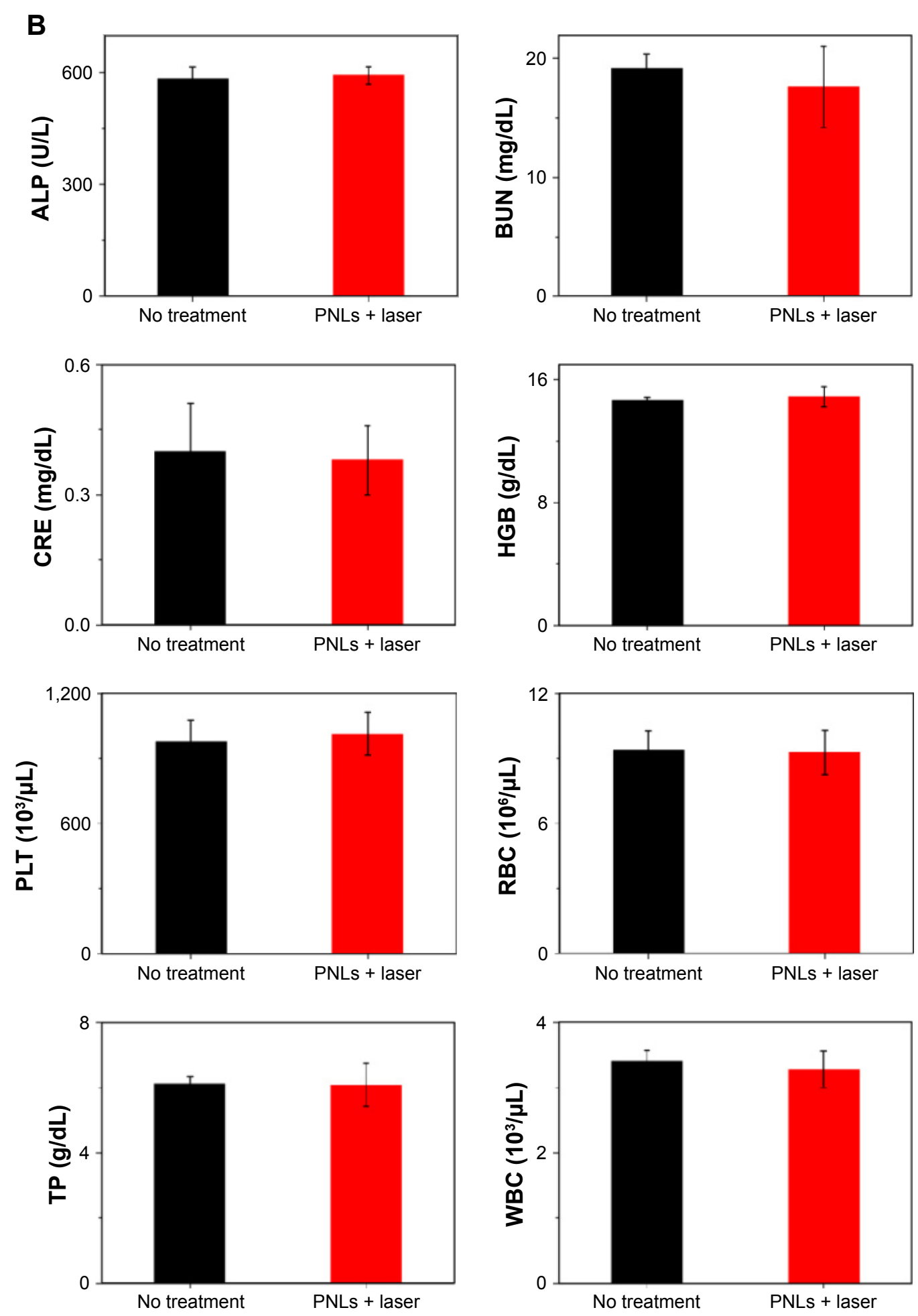

Figure 8 In vivo biosafety studies.

Notes: (A) TUNEL staining images of heart tissues in mice with DOX and PNLs + laser treatments for 15 days; scale bar represents $200 \mu \mathrm{m}$. (B) The complete blood panel data from PNLs + laser-treated mice and no treatment group: RBC, WBC, PLT, TP, ALP, BUN, CRE, and HGB. Data presented are mean \pm SD ( $n=3$ ).

Abbreviations: ALP, alkaline phosphatase; BUN, blood urea nitrogen; CRE, creatinine; HGB, hemoglobin; DOX, doxorubicin; PLT, platelet; PNLs, photo-activated nanoliposomes; RBC, red blood cell; TP, total protein; WBC, white blood cell.

drug release and photo-controlled PDT) was prepared and investigated in vitro and in vivo. We surmised that PNLs can combine the strengths of long blood circulation times, tumor-targeting ability and superior chemotherapeutic efficacy of DOX, and the potent PDT efficacy of HMME in the single photo-activated platform to afford maximal anticancer efficacy with low drug doses for the treatment of breast-resistant tumors. 
The EE of the water-soluble drug DOX (in the inner hydrophilic core) and the lipophilic PS HMME (in the hydrophobic lipid layer) in PNLs were $\sim 58.8$ and $\sim 43.7 \%$, respectively. These results showed that PNLs had a remarkable capability of delivering large amounts of both chemotherapeutic agents and PSs. More importantly, PNLs released its payloads in an obvious photo-triggered manner. This means that when there is no laser irradiation, PNLs could efficiently maintain DOX and HMME, allowing high delivery efficiency and tumor accumulation of both DOX and HMME in one platform. Interestingly, with the HMME inserting into hydrophobic lipid layer of PNLs, the release of DOX was much slower, indicating that the loading of HMME maybe enhance the stability and compactness of the liposome, thus decreasing the unexpected DOX release during circulation. The high spatial/temporal resolution photo-triggered DOX and HMME release manner of PNLs was also expected to bring more benefits for the treatment of tumors.

Small-molecule drugs typically suffer from nonspecific distribution throughout the body, rapid clearance, and low accumulation at the tumor site when given systemically. ${ }^{43}$ While, the PEG-modified liposome can enhance tumor uptake of drugs via the EPR effect. ${ }^{44,45}$ After intravenous injection to tumor bearing mice, PNLs exhibited a significant tumortargeting property. This excellent tumor-targeting ability of PNLs can be attributed to the small particle size $(\sim 120 \mathrm{~nm})$, PEG coating, and favorable structural stability of PNLs in extracellular environments. As a result, PNLs achieved a high accumulation in the tumor $4 \mathrm{~h}$ post intravenous injection and with low uptake by the MPS system and minimal nonspecific organ distributions.

PNLs not only exhibited efficient and highly specific tumor deposition but also showed high uptake in the cancer cells. After incubated with MCF-7/MDR cells for $4 \mathrm{~h}$, the cellular uptake amount of PNLs was much more than that of DOX (with the same DOX concentration). Meanwhile, the photo-responsive drug release ability of PNLs was still efficient in the cultured MCF-7/MDR cells. More importantly, after laser irradiation, negligible efflux of DOX was observed for PNLs in MCF-7/MDR cells throughout the next $4 \mathrm{~h}$ incubation period due to the significant decrease of P-gp in MCF-7/MDR cells. The large amount of generated ROS from PNLs upon laser irradiation could be partly responsible for the negligible DOX efflux from cancer cells. The results were observed in both the in vitro and in vivo studies.

Recently, combination therapy is more and more popular in cancer therapy; for example, photothermalchemotherapy, ${ }^{32,46}$ photodynamic-chemotherapy, ${ }^{5,47}$ and combination therapy always display higher cancer treatment efficiency than any single treatment. Combination therapy offers the opportunities to treat the cancers through different mechanisms of actions, thus leading to enhanced anticancer efficacy via synergistic effects. PNLs combined the superior chemotherapy efficacy of DOX and potent PDT efficacy of HMME in one single platform and significantly enhanced the anticancer efficacy in DOX-resistant breast tumor both in vitro and in vivo. This synergistic effect was substantiated by the following results: 1) significantly increased DOX or HMME antitumor efficacy of PNLs with laser irradiation compared to DOX, NL(DOX), NL(HMME), NL(DOX) + NL(HMME) + laser, or PNLs without laser irradiation in the in vivo antitumor effect tests, the tumors of mice receiving PNLs and irradiation shrank by $\sim 82 \%$ in volume; and 2 ) significantly decreased the level of P-gp of PNLs with laser irradiation compared to the no treatment, laser irradiation alone, PNLs without laser irradiation group, the level of P-gp of PNLs-treated mice decreased to $23.6 \%$ (normalized to the no treatment control group 100\%). We have thus demonstrated that PNLs shrink the drug-resistant breast tumors in mouse xenograft models via intravenous administration of nanoparticles carrying both DOX and HMME.

Besides the combination therapeutic effect, PNLs significantly decreased the side effect of DOX. After treatment with DOX $(5 \mathrm{mg} / \mathrm{kg}$ ) for 15 days, a severe cardiac toxicity was observed, while negligible cardiac toxicity was observed in PNL-related groups. Dose-limiting side effects prevent complete eradication of most cancers. Intolerable high doses of therapeutics are needed to achieve effective anticancer efficacy owing to the nonideal biodistribution of most drugs. ${ }^{48}$ In this study, PNLs significantly improved the tumor accumulation and decreased nonspecific organ distributions of DOX and HMME after intravenous injection, thus allowing enhanced anticancer efficacy with little side effects. The high biosafety of PNLs with laser irradiation was substantiated by the complete blood panel data analysis. Importantly, energy irradiance and irradiation time for PDT were $300 \mathrm{~mW} / \mathrm{cm}^{2}$ and $15 \mathrm{~min}$, respectively, which are comparable to clinical parameters for PDT, thus leading to no skin/tissue damage in the irradiated region.

In this study, through a combination of nanotechnology and photo-controlled drug release and a combination of chemotherapy and PDT, PNLs thus open a window for achieving maximal anticancer efficacy with minimal side effects in drug-resistant breast tumor-bearing mice.

In conclusion, in this study, we have developed a liposome-based PNL that combines two treatment modalities, chemotherapy and PDT, into one single platform to 
allow potent anticancer activity in drug-resistant breast cancers. PNLs have several distinctive capabilities: 1) carrying high loadings of DOX and HMME and releasing the payloads in a photo-cleavage manner with high spatial/ temporal resolution at the site of actions via photocatalysis; 2) reducing DOX efflux in MCF-7/MDR cells via decreasing the level of P-gp induced by PDT; 3) accumulating in tumor site taking advantage of the EPR effect; and 4) combining effective chemotherapy and PDT to exert much enhanced anticancer effect and achieving significant tumor regression in a drug-resistant tumor model with little side effects. PNLs provide a general platform for incorporating multiple therapeutic agents or/and modalities for treating many cancers, including drug-resistant cancers. As the synthesis of PNLs is very simple and highly scalable, PNLs offer a versatile and effective DDS for potential translation to the clinic.

\section{Acknowledgments}

The work is supported by grants from the National Natural Science Foundation of China (nos 81601597 and 81302717), key scientific research projects (Education Department of Henan Province, no 17A350003) and Postdoctoral Science Foundation of China (no 2015M582210).

\section{Disclosure}

The authors report no conflicts of interest in this work.

\section{References}

1. Ruoslahti E. Tumor penetrating peptides for improved drug delivery. Adv Drug Deliv Rev. 2017;110-111:3-12.

2. Shi J, Wang B, Wang L, et al. Fullerene (C60)-based tumor-targeting nanoparticles with "off-on" state for enhanced treatment of cancer. J Control Release. 2016;235:245-258.

3. Xin Y, Huang Q, Tang JQ, et al. Nanoscale drug delivery for targeted chemotherapy. Cancer Lett. 2016;379(1):24-31.

4. Yang Q, Li L, Sun W, Zhou Z, Huang Y. Dual stimuli-responsive hybrid polymeric nanoparticles self-assembled from POSS-based starlike copolymer-drug conjugates for efficient intracellular delivery of hydrophobic drugs. ACS Appl Mater Interfaces. 2016;8(21): 13251-13261.

5. Shi J, Chen Z, Wang L, et al. A tumor-specific cleavable nanosystem of PEG-modified C60@Au hybrid aggregates for radio frequencycontrolled release, hyperthermia, photodynamic therapy and X-ray imaging. Acta Biomater. 2016;29:282-297.

6. Pang X, Jiang Y, Xiao Q, Leung AW, Hua H, Xu C. pH-responsive polymer-drug conjugates: design and progress. J Control Release. 2016;222:116-129.

7. Wang M, Li J, Li X, et al. Magnetically and $\mathrm{pH}$ dual responsive dendrosomes for tumor accumulation enhanced folate-targeted hybrid drug delivery. J Control Release. 2016;232:161-174.

8. Koetting MC, Guido JF, Gupta M, Zhang A, Peppas NA. pH-responsive and enzymatically-responsive hydrogel microparticles for the oral delivery of therapeutic proteins: effects of protein size, crosslinking density, and hydrogel degradation on protein delivery. J Control Release. 2016;221:18-25.
9. Yang P, Li D, Jin S, et al. Stimuli-responsive biodegradable poly(methacrylic acid) based nanocapsules for ultrasound traced and triggered drug delivery system. Biomaterials. 2014;35(6):2079-2088.

10. Wei H, Zhang XZ, Zhou Y, Cheng SX, Zhuo RX. Self-assembled thermoresponsive micelles of poly(N-isopropylacrylamide-b-methyl methacrylate). Biomaterials. 2006;27(9):2028-2034.

11. Kang H, Trondoli AC, Zhu G, et al. Near-infrared light-responsive core-shell nanogels for targeted drug delivery. ACS Nano. 2011; 5(6):5094-5099.

12. Feng X, Jiang D, Kang T, et al. Tumor-homing and penetrating peptidefunctionalized photosensitizer-conjugated PEG-PLA nanoparticles for chemo-photodynamic combination therapy of drug-resistant cancer. ACS Appl Mater Interfaces. 2016;8(28):17817-17832.

13. Carregal-Romero S, Ochs M, Rivera-Gil P, et al. NIR-light triggered delivery of macromolecules into the cytosol. J Control Release. 2012; 159(1):120-127.

14. Hühn D, Govorov A, Gil PR, Parak WJ. Photostimulated Au nanoheaters in polymer and biological media: characterization of mechanical destruction and boiling. Adv Funct Mater. 2012;22(2):294-303.

15. Rivera Gil P, Huhn D, del Mercato LL, Sasse D, Parak WJ. Nanopharmacy: inorganic nanoscale devices as vectors and active compounds. Pharmacol Res. 2010;62(2):115-125.

16. Spring BQ, Bryan Sears R, Zheng LZ, et al. A photoactivable multiinhibitor nanoliposome for tumour control and simultaneous inhibition of treatment escape pathways. Nat Nanotechnol. 2016;11(4):378-387.

17. Yang D, Wang T, Su Z, Xue L, Mo R, Zhang C. Reversing cancer multidrug resistance in xenograft models via orchestrating multiple actions of functional mesoporous silica nanoparticles. ACS Appl Mater Interfaces. 2016;8(34):22431-22441.

18. Li D, Zhou L, Huang J, Xiao X. Effect of multidrug resistance 1/Pglycoprotein on the hypoxia-induced multidrug resistance of human laryngeal cancer cells. Oncol Lett. 2016;12(2):1569-1574.

19. Dai Z, Yao Q, Zhu L. MMP2-sensitive PEG-lipid copolymers: a new type of tumor-targeted P-glycoprotein inhibitor. ACS Appl Mater Interfaces. 2016;8(20):12661-12673.

20. Fletcher JI, Williams RT, Henderson MJ, Norris MD, Haber M. ABC transporters as mediators of drug resistance and contributors to cancer cell biology. Drug Resist Updat. 2016;26:1-9.

21. Chen Z, Shi T, Zhang L, et al. Mammalian drug efflux transporters of the ATP binding cassette $(\mathrm{ABC})$ family in multidrug resistance: a review of the past decade. Cancer Lett. 2016;370(1):153-164.

22. Wu RW, Chu ES, Huang Z, Xu CS, Ip CW, Yow CM. Effect of FosPeg $(\mathrm{R})$ mediated photoactivation on $\mathrm{P}-\mathrm{gp} / \mathrm{ABCB} 1$ protein expression in human nasopharyngeal carcinoma cells. $J$ Photochem Photobiol B. 2015;148:82-87.

23. Cao W, Zeng X, Liu G, et al. Porphine functionalized nanoparticles of star-shaped poly(epsilon-caprolactone)-b-D-alpha-tocopheryl polyethylene glycol 1000 succinate biodegradable copolymer for chemophotodynamic therapy on cervical cancer. Acta Biomater. 2015;26: $145-158$.

24. Wong BS, Yoong SL, Jagusiak A, et al. Carbon nanotubes for delivery of small molecule drugs. Adv Drug Deliv Rev. 2013;65(15):1964-2015.

25. Tham HP, Chen H, Tan YH, et al. Photosensitizer anchored gold nanorods for targeted combinational photothermal and photodynamic therapy. Chem Commun (Camb). 2016;52(57):8854-8857.

26. Zhuang X, Ma X, Xue X, et al. A photosensitizer-loaded DNA origami nanosystem for photodynamic therapy. ACS Nano. 2016;10(3): 3486-3495.

27. Kolemen S, Ozdemir T, Lee D, et al. Remote-controlled release of singlet oxygen by the plasmonic heating of endoperoxide-modified gold nanorods: towards a paradigm change in photodynamic therapy. Angew Chem Int Ed Engl. 2016;55(11):3606-3610.

28. Chen HP, Tung FI, Chen MH, Liu TY. A magnetic vehicle realized tumor cell-targeted radiotherapy using low-dose radiation. J Control Release. 2016;226:182-192.

29. Liu D, Yang F, Xiong F, Gu N. The smart drug delivery system and its clinical potential. Theranostics. 2016;6(9):1306-1323. 
30. Brown PK, Qureshi AT, Moll AN, Hayes DJ, Monroe WT. Silver nanoscale antisense drug delivery system for photoactivated gene silencing. ACS Nano. 2013;7(4):2948-2959.

31. Chen C, Zhou L, Geng J, Ren J, Qu X. Photosensitizer-incorporated quadruplex DNA-gated nanovechicles for light-triggered, targeted dual drug delivery to cancer cells. Small. 2013;9(16):2793-2800.

32. Shi J, Wang L, Zhang J, et al. A tumor-targeting near-infrared lasertriggered drug delivery system based on GO@Ag nanoparticles for chemo-photothermal therapy and X-ray imaging. Biomaterials. 2014; 35(22):5847-5861.

33. McCoy CP, Rooney C, Edwards CR, Jones DS, Gorman SP. Lighttriggered molecule-scale drug dosing devices. J Am Chem Soc. 2007; 129(31):9572-9573.

34. Chen J, Guo Z, Wang HB, et al. MultifunctionalFe3O4@C@Aghybrid nanoparticles as dual modal imaging probes and near-infrared lightresponsive drug delivery platform. Biomaterials. 2013;34(2):571-581.

35. Perrie Y, Crofts F, Devitt A, Griffiths HR, Kastner E, Nadella V. Designing liposomal adjuvants for the next generation of vaccines. Adv Drug Deliv Rev. 2016;99(pt A):85-96.

36. Chen ZL, Huang M, Wang XR, et al. Transferrin-modified liposome promotes alpha-mangostin to penetrate the blood-brain barrier. Nanomedicine. 2016;12(2):421-430.

37. Alshaer W, Hillaireau H, Vergnaud J, Ismail S, Fattal E. Functionalizing liposomes with anti-CD44 aptamer for selective targeting of cancer cells. Bioconjug Chem. 2015;26(7):1307-1313.

38. Koudelka S, Turanek Knotigova P, Masek J, et al. Liposomal delivery systems for anti-cancer analogues of vitamin E. J Control Release. 2015;207:59-69.
39. Dahal E, Curtiss J, Subedi D, Chen G, Houston JP, Smirnov S. Evaluation of the catalytic activity and cytotoxicity of palladium nanocubes: the role of oxygen. ACS Appl Mater Interfaces. 2015;7(18):9364-9371.

40. Wang M, Kim JC. Light- and temperature-responsive liposomes incorporating cinnamoyl Pluronic F127. Int J Pharm. 2014; 468(1-2):243-249.

41. Bikram M, West JL. Thermo-responsive systems for controlled drug delivery. Expert Opin Drug Deliv. 2008;5(10):1077-1091.

42. He C, Liu D, Lin W. Self-assembled core-shell nanoparticles for combined chemotherapy and photodynamic therapy of resistant head and neck cancers. ACS Nano. 2015;9(1):991-1003.

43. Della Rocca J, Liu D, Lin W. Nanoscale metal-organic frameworks for biomedical imaging and drug delivery. Acc Chem Res. 2011; 44(10):957-968.

44. Peer D, Karp JM, Hong S, Farokhzad OC, Margalit R, Langer R. Nanocarriers as an emerging platform for cancer therapy. Nat Nanotechnol. 2007;2(12):751-760.

45. Gil PR, Parak WJ. Composite nanoparticles take aim at cancer. ACS Nano. 2008;2(11):2200-2205.

46. Shi J, Wang L, Gao J, et al. A fullerene-based multi-functional nanoplatform for cancer theranostic applications. Biomaterials. 2014; 35(22):5771-5784.

47. Shi J, Zhang H, Wang L, et al. PEI-derivatized fullerene drug delivery using folate as a homing device targeting to tumor. Biomaterials. 2013; 34(1):251-261.

48. Torchilin V. Tumor delivery of macromolecular drugs based on the EPR effect. Adv Drug Deliv Rev. 2011;63(3):131-135. 


\section{Supplementary materials}
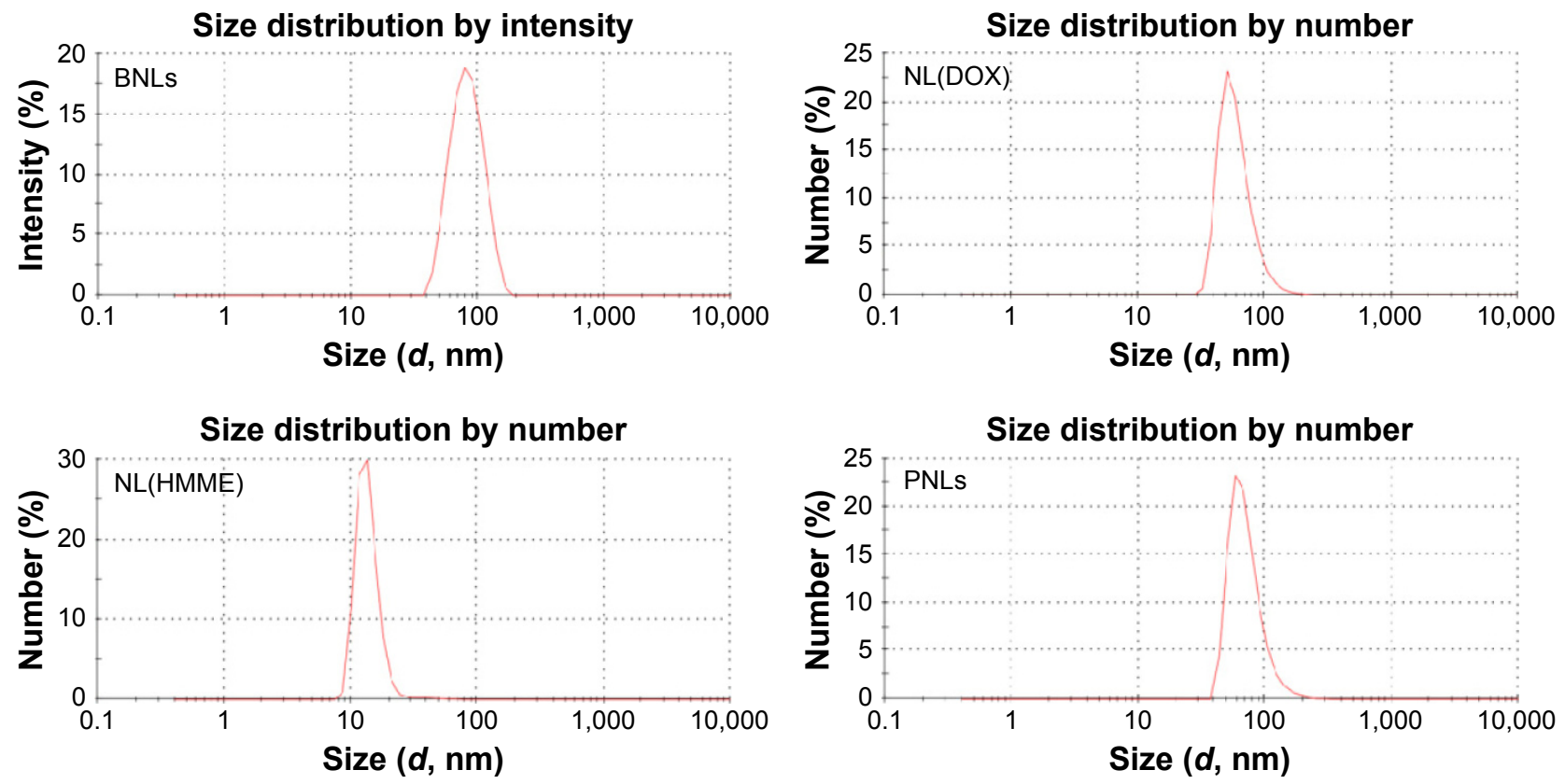

Figure SI DLS results of BNLs, NL(DOX), NL(HMME), and PNLs in water.

Abbreviations: BNLs, blank nanoliposomes; DOX, doxorubicin; HMME, hematoporphyrin monomethyl ether; NL(DOX), DOX-loaded nanoliposome; NL(HMME), HMMEloaded nanoliposome; PNLs, photo-activated nanoliposomes.

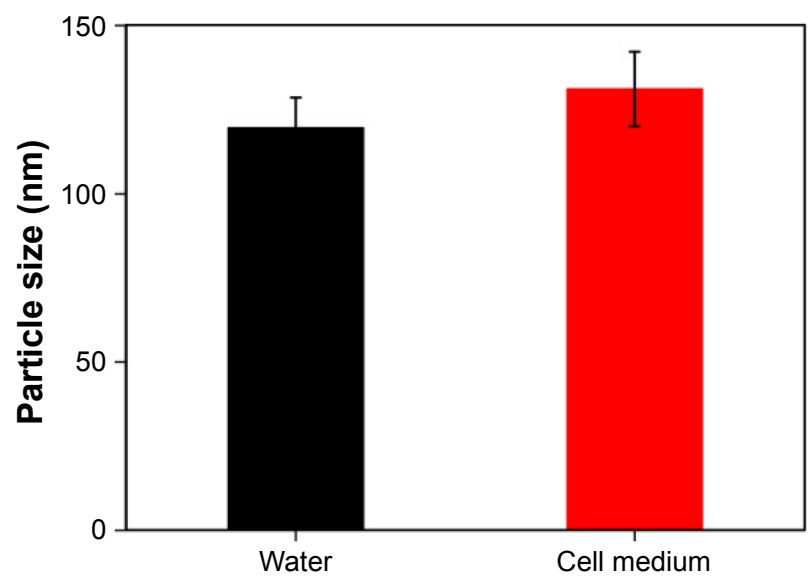

Figure S2 Particle size of PNLs in water and cell medium for $12 \mathrm{~h}$. Note: Data presented are mean $\pm S D(n=3)$.

Abbreviation: PNLs, photo-activated nanoliposomes.

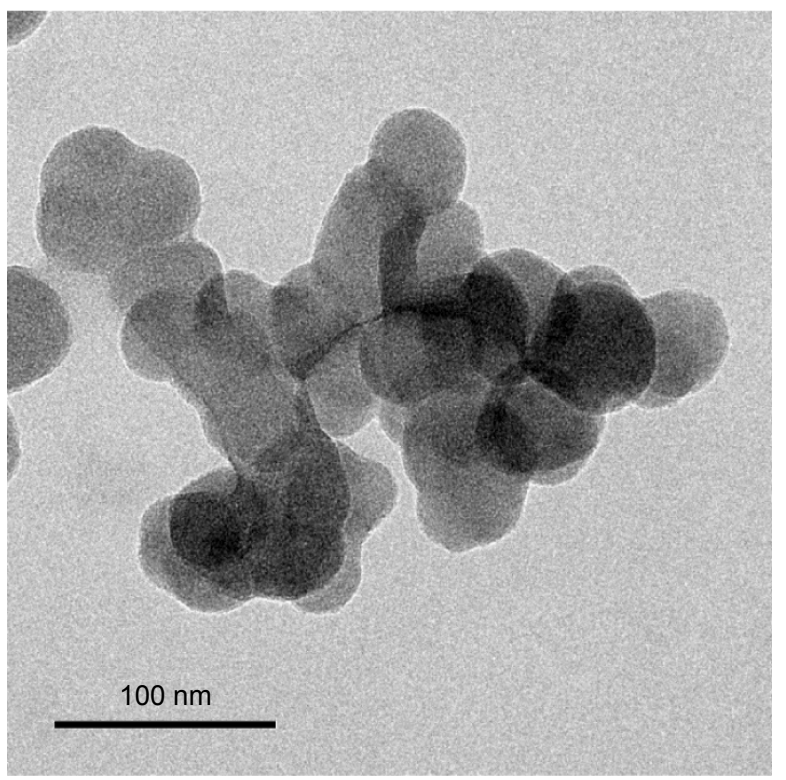

Figure S3 TEM image of PNLs with high concentration (diluted 10 times). Abbreviations: PNLs, photo-activated nanoliposomes; TEM, transmission electron microscope. 


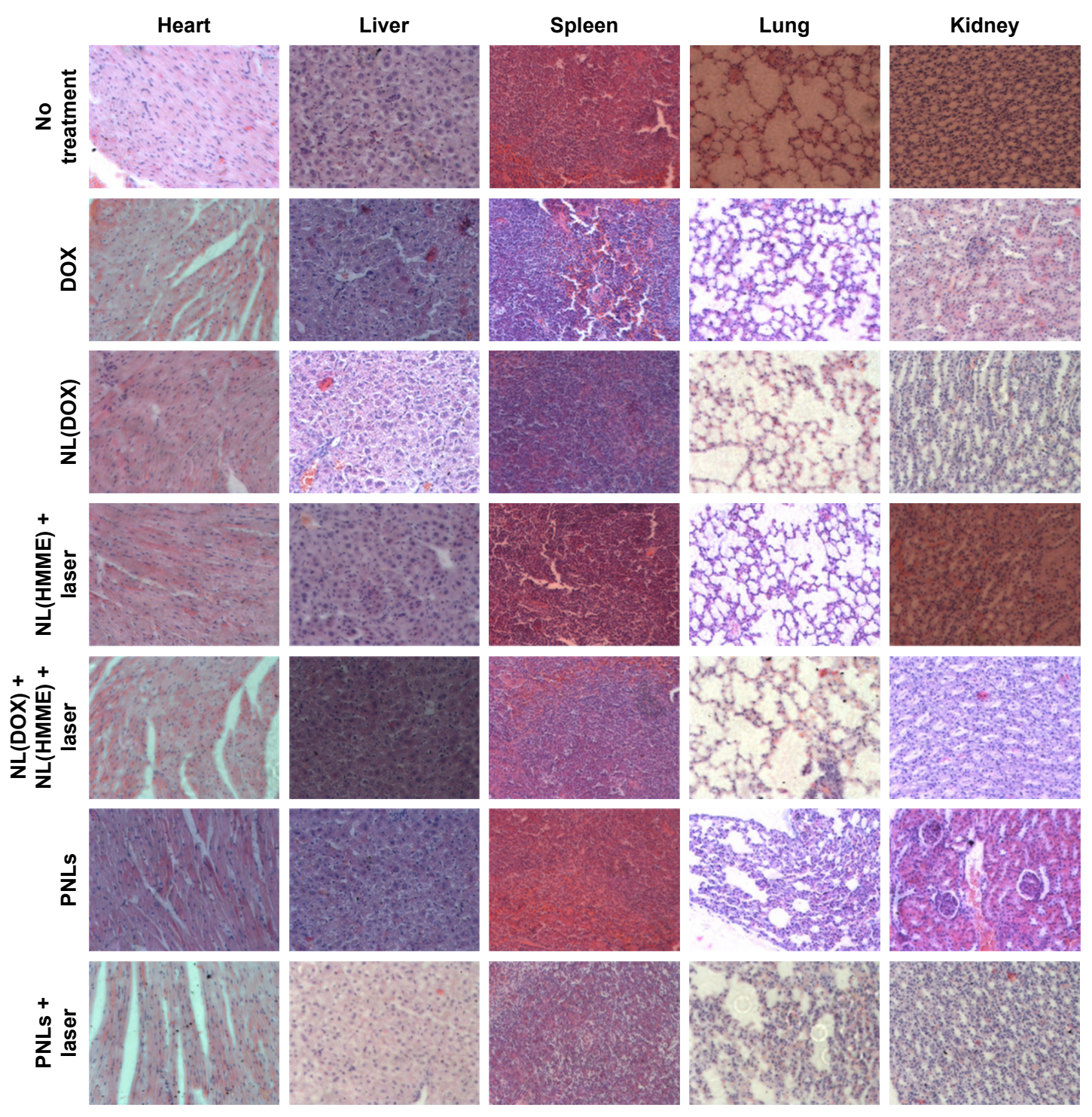

Figure S4 HE staining images of heart, liver, spleen, lung, and kidney tissues in mice with different treatments for 15 days.

Abbreviations: DOX, doxorubicin; HMME, hematoporphyrin monomethyl ether; NL(DOX), DOX-loaded nanoliposome; NL(HMME), HMME-loaded nanoliposome; PNLs, photo-activated nanoliposomes.

International Journal of Nanomedicine

\section{Publish your work in this journal}

The International Journal of Nanomedicine is an international, peerreviewed journal focusing on the application of nanotechnology in diagnostics, therapeutics, and drug delivery systems throughou the biomedical field. This journal is indexed on PubMed Central,

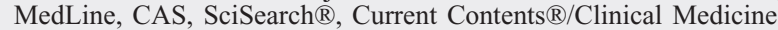

\section{Dovepress}

Journal Citation Reports/Science Edition, EMBase, Scopus and the Elsevier Bibliographic databases. The manuscript management system is completely online and includes a very quick and fair peer-review system, which is all easy to use. Visit http://www.dovepress.com/ testimonials.php to read real quotes from published authors.

\footnotetext{
Submit your manuscript here: http://www.dovepress.com/international-journal-of-nanomedicine-journal
} 